\title{
Review
}

Molecular

Neuropsychiatry
Mol Neuropsychiatry 2016;2:61-78

DOI: 10.1159/000445802
Received: November 23, 2015

Accepted: March 24, 2016

Published online: May 20, 2016

\section{Genetics of Common Antipsychotic-Induced Adverse Effects}

\author{
Raymond R. MacNeila Daniel J. Müller ${ }^{b-d}$ \\ a Mood Research Laboratory, Department of Psychology, Queen's University, Kingston, Ont., Departments of \\ ${ }^{\mathrm{b}}$ Psychiatry, and 'Pharmacology and Toxicology, University of Toronto, and 'Pharmacogenetics Research Clinic, \\ Campbell Family Mental Health Research Institute, Centre for Addiction and Mental Health, Toronto, Ont., Canada
}

\section{Key Words}

Antipsychotic · Adverse effect - Extrapyramidal symptoms .

Genetic association study - Metabolic syndrome .

Pharmacogenetics · Polymorphism - Tardive dyskinesia .

Weight gain

\begin{abstract}
The effectiveness of antipsychotic drugs is limited due to accompanying adverse effects which can pose considerable health risks and lead to patient noncompliance. Pharmacogenetics ( $P G x$ ) offers a means to identify genetic biomarkers that can predict individual susceptibility to antipsychoticinduced adverse effects (AAEs), thereby improving clinical outcomes. We reviewed the literature on the PGx of common AAEs from 2010 to 2015, placing emphasis on findings that have been independently replicated and which have additionally been listed to be of interest by PGx expert panels. Gene-drug associations meeting these criteria primarily pertain to metabolic dysregulation, extrapyramidal symptoms (EPS), and tardive dyskinesia (TD). Regarding metabolic dysregulation, results have reaffirmed $H T R 2 C$ as a strong candidate with potential clinical utility, while $M C 4 R$ and OGFR1 gene loci have emerged as new and promising biomarkers for the prediction of weight gain. As for EPS and TD, additional evidence has accumulated in support of an asso-
\end{abstract}

ciation with CYP2D6 metabolizer status. Furthermore, HSPG2 and DPP6 have been identified as candidate genes with the potential to predict differential susceptibility to TD. Overall, considerable progress has been made within the field of psychiatric PGx, with inroads toward the development of clinical tools that can mitigate AAEs. Going forward, studies placing a greater emphasis on multilocus effects will need to be conducted.

(c) 2016 S. Karger AG, Basel

\section{Introduction}

Antipsychotic (AP) drugs are the mainstay pharmacological treatment for schizophrenia (SCZ) and related psychotic disorders. APs have also been shown to be effective for the treatment of other psychiatric conditions, including bipolar disorder, treatment-resistant depression, and autism spectrum disorders (ASDs) [1-3]. Despite their demonstrable clinical utility, significant interindividual variation in the therapeutic efficacy and tolerability of APs presents a significant challenge for physicians and their patients.

The introduction of second-generation 'atypical' APs (SGAs; e.g., risperidone) was highly welcomed by the psychiatric community, as these drugs were thought to rep-

\section{KARGER}

E-Mail karger@karger.com www.karger.com/mnp (c) 2016 S. Karger AG, Base

2296-9209/16/0022-0061\$39.50/0
Daniel J. Müller, MD, PhD

Centre for Addiction and Mental Health

250 College Street, R132

Toronto, ON M5T 1R8 (Canada)

E-Mail daniel.mueller@ camh.ca 
resent a significant improvement over first-generation 'typical' APs (FGAs; e.g., haloperidol). The use of FGAs had been marred by the tendency of these drugs to cause extrapyramidal symptoms (EPS), and with long-term use, tardive dyskinesia (TD), a highly debilitating and potentially irreversible movement disorder $[4,5]$. While the risk of developing EPS and TD associated with SGAs is substantially lower, these adverse effects (AEs) still represent an ongoing concern $[6,7]$. Moreover, the use of SGAs is often accompanied by weight gain and related cardiometabolic abnormalities, thereby putting patients at a greater risk of developing diabetes and cardiovascular disease [8-10]. Importantly, whether caused by FGAs or SGAs, AP-induced AEs (AAEs) are a major source of patient noncompliance and treatment discontinuation, both of which lead to greater functional impairment, higher rates of relapse, and an increased risk of suicide [11-14]. In view of this, dissecting the underlying factors contributing to interindividual variation in susceptibility to AAEs may facilitate better treatment selection, and thereby improve clinical outcomes.

Pharmacogenetics (PGx) is a field of study and clinical tool that assesses how genetic variability influences drug response and tolerability. Evidence from twin studies implicates a significant genetic component underlying individual differences in susceptibility to AAEs $[15,16]$. Therefore, PGx has the potential to significantly improve the treatment of SCZ and other neuropsychiatric disorders. Considering the progress of PGx in the last two decades, it seems inevitable that pharmacogenetic testing accounting for genetic variability in both pharmacokinetic and pharmacodynamic processes - will eventually play a role in the prescription of APs and other psychotropic drugs. It is hoped that physicians will be able to utilize information from pharmacogenetic tests in order to deliver personalized drug therapies with optimal efficacy and minimal AEs. Although still in the early stages of their predictive capacity, several psychiatric-based PGx tests have already begun to be offered by private companies [for a review, see 17]. Furthermore, several clinical PGx expert panels, such as the Pharmacogenomics Knowledgebase (PharmGKB) and the Clinical Pharmacogenetics Implementation Consortium (CPIC) [18-20], as well as drug regulatory agencies (e.g., US Food and Drug Administration) [21, 22], have begun to propose guidelines to assist physicians in interpreting the clinical significance of information provided by these tests. Finally, recent studies assessing the feasibility and utility of incorporating $\mathrm{PGx}$ into standard psychiatric practice have yielded encouraging results [23-27].
Here, we survey the literature published on the PGx of AAEs within the last 5 years (2010-2015), placing emphasis on drug-gene associations replicated in independent samples and which have additionally been listed by expert panels as having potential clinical relevance. Several highquality reviews providing extensive coverage on the AP PGx literature published prior to 2010 are available [2831].

\section{Methods}

PubMed, Embase (Ovid), and PsycINFO (Ovid) databases were searched using the following combination of key-terms and/ or their matched subject headings: ('antipsychotic' OR 'neuroleptic') AND ('pharmacogenetics' OR 'pharmacogenomics' OR 'genetic association study' OR 'polymorphism') AND ('adverse effect' OR 'tardive dyskinesia' OR 'extrapyramidal symptoms' OR 'weight gain'). Our search parameters limited results to include only peerreviewed articles that were published in English from 2010 to 2015 inclusive. Letters to the editor, editorials, and publications solely related to drug efficacy, or which were otherwise irrelevant to the subject of our review were excluded. The reference lists of retained publications were also screened for other relevant studies. Given the abundance of literature available, emphasis has been placed on independently replicated gene-drug relationships of common AAEs that have additionally been recognized by expert panels as having potential clinical relevance.

\section{Results}

Studies meeting our criteria represent two major categories of AAEs: (1) metabolic dysregulation, including weight gain and/or metabolic syndrome (MetS), and (2) movement disorders, including EPS and TD. In total, we have reviewed 53 studies that report on variants within 11 different genes.

\section{Weight Gain and MetS}

AP-induced weight gain (AIWG) has an incidence of approximately $30 \%$ and is most pronounced among patients treated with the SGAs clozapine and olanzapine [32]. The heritability $\left(\mathrm{h}^{2}\right)$ of AIWG is estimated to be $0.6-$ 0.8 [16]. AIWG often coincides with the development of MetS, a complex phenotype characterized by central obesity, insulin resistance, hyperglycemia, dyslipidemia, and hypertension [8]. With respect to AIWG and AP-induced MetS (AP-MetS), we report on variants within genes HTR2C, LEP, LEPR, MC4R, MTHFR, and OGFRL1. Information on gene-drug interactions listed to be of potential clinical significance by expert PGx panels is given in
MacNeil/Müller 
Table 1. Putative genetic associations with common metabolic AAEs studied from 2010 to 2015 and their related listings by expert PGx panels

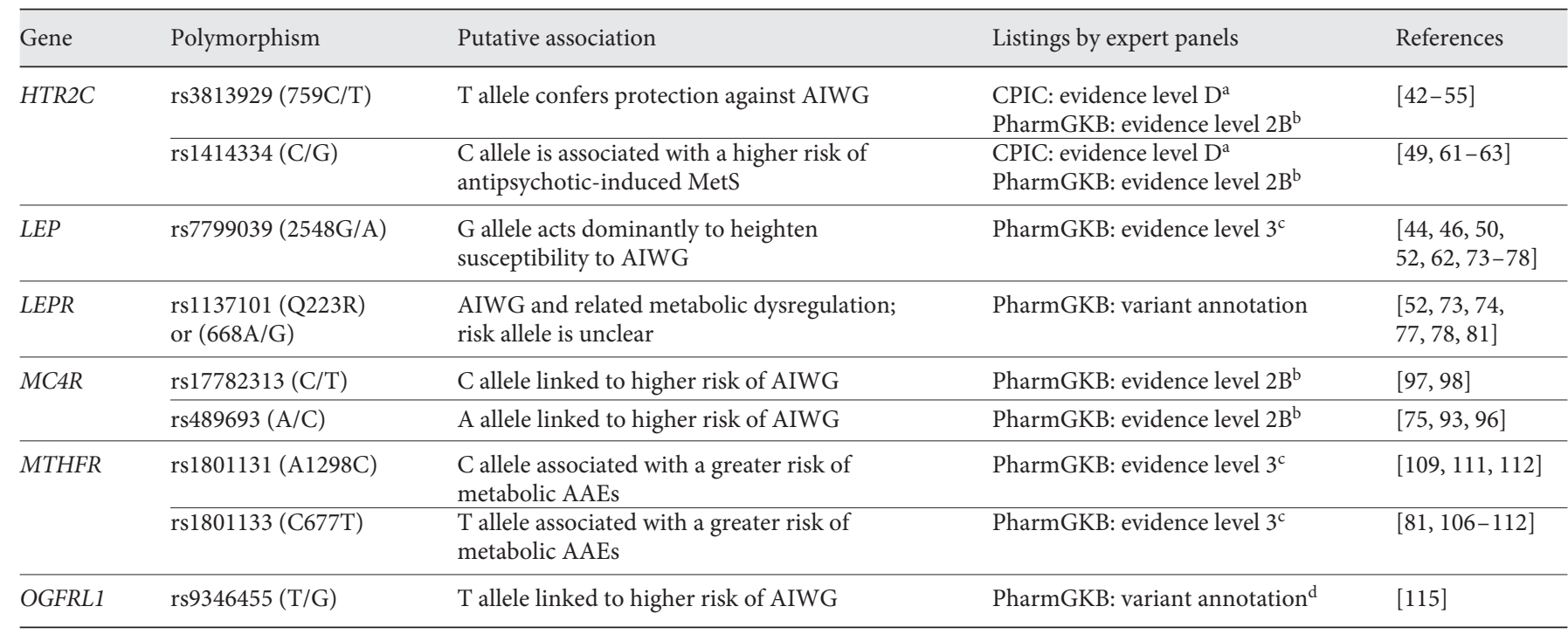

References include both positive and negative findings. See references $[19,20]$ for further information on the PharmGKB and CPIC, as well as the criteria used to assign evidence levels. ${ }^{\text {a }}$ Considered of interest. ${ }^{\mathrm{b}}$ Moderate evidence for this association. ${ }^{\mathrm{c}}$ Low evidence for this association. ${ }^{\mathrm{d}}$ Listed under IBA57.

table 1. A detailed overview of the studies, including information on design, treatment duration, AP use, sample demographics, main results, and associated odds ratios and/or $\mathrm{p}$ values (if applicable), is provided in table 2 .

\section{HTR2C}

Serotonin $2 \mathrm{C}$ receptors $\left(5-\mathrm{HT}_{2 \mathrm{C}} \mathrm{Rs}\right)$ are integral to the regulation of energy homeostasis via their interaction with the melanocortin and leptin signaling pathways [3335]. The most potent weight-inducing APs are characterized by their high affinity for $5-\mathrm{HT}_{2 \mathrm{C}} \mathrm{Rs}$ and the antagonistic properties they have at these receptors [36]. 5$\mathrm{HT}_{2 \mathrm{C}} \mathrm{R}$ antagonism promotes hyperphagic activity and attenuates energy expenditure [35]. In light of this, the $\mathrm{X}$-linked HTR2C gene encoding $5-\mathrm{HT}_{2 \mathrm{C}} \mathrm{Rs}$ has been among the most extensively investigated genes with respect to AP-induced metabolic dysregulation. Results from studies conducted prior to 2010 have pointed to a consistent relationship between the $\mathrm{rs} 3813929(-759 \mathrm{C} / \mathrm{T})$ promoter SNP and AIWG $[28,37]$. This SNP has been shown to affect the transcription of HTR2C, though the exact impact on gene expression remains unclear [3841].

Several studies included in our results have replicated the association of $-759 \mathrm{C} / \mathrm{T}$ with AIWG [42-46], though negative and contradictory results were also reported [47-54]. One study involving a heterogeneous psychiat- ric sample reported that carriers of the HTR2C $-759 \mathrm{~T}$ allele were protected against the weight-inducing effects of olanzapine [42]. This association was also confirmed in a pediatric ASD sample treated with risperidone [43]. However, 2 other studies also involving samples of pediatric patients with ASDs could not verify this result [47, 48]. Opgen-Rhein et al. [44] had initially identified a nominally significant association between the $-759 \mathrm{C} / \mathrm{T}$ SNP and AIWG. However, a haplotype analysis revealed that the A-G-C haplotype (of SNPs rs498207, rs3813928, and rs3813929, respectively) was significantly overrepresented in the weight gain group (i.e. $\geq 7 \%$ increase in body weight), while the opposite haplotype (G-A-T) was overrepresented in the control group.

A study by Sicard et al. [45] provides additional support for the involvement of $-759 \mathrm{C} / \mathrm{T}$ in differential susceptibility to AIWG. Haplotype analyses including correlated SNPs rs518147 (G-697C) and rs6318 (Ser23Cys) showed that the C-G-Cys23 haplotype was significantly overrepresented within the weight gain group. Furthermore, a meta-analysis published as part of the same study confirmed the association between the HTR2C $-759 \mathrm{~T}$ allele and resistance to AIWG. The effect size was more pronounced when limiting the analysis to studies including all or almost all European subjects and excluding Asian samples. Limiting the analysis to samples with all or almost all European subjects also eliminated signifi- 
Table 2. Overview of studies and their main findings related antipsychotic-induced weight gain and/or metabolic syndrome

\begin{tabular}{|c|c|c|c|c|c|}
\hline Gene & $\begin{array}{l}\text { Poly- } \\
\text { morphism(s) }\end{array}$ & $\begin{array}{l}\text { Design, } \\
\text { treatment } \\
\text { duration }\end{array}$ & Medication (n) & $\begin{array}{l}\text { Sample [diagnosis; } \mathrm{n}(\mathrm{m} / \mathrm{f}) \text {; } \\
\text { ethnicity/race] }\end{array}$ & Main findings \\
\hline HTR2C & $\begin{array}{l}\text { rs3813929 } \\
(759 \mathrm{C} / \mathrm{T})\end{array}$ & $\begin{array}{l}\text { prospective } \\
4 \text { weeks }\end{array}$ & olanzapine (124) & $\begin{array}{l}\text { psychotic or mood disorder; } n=124 \\
(62 / 62) ; \text { German-Caucasian }(124)\end{array}$ & $\begin{array}{l}\text { olanzapine treatment associated with change } \\
\text { in BMI }(\mathrm{p}=0.009) \text { and weight }(\mathrm{p}=0.008) \\
{[42]}\end{array}$ \\
\hline HTR2C & $\begin{array}{l}\text { rs3813929 } \\
(759 \mathrm{C} / \mathrm{T})\end{array}$ & $\begin{array}{l}\text { retrospective } \\
8 \text { weeks }\end{array}$ & risperidone (36) & $\begin{array}{l}\text { ASD pediatric sample ( } 5-16 \text { years); } \\
\mathrm{n}=32(28 / 4) ; \text { all Caucasian }\end{array}$ & $\begin{array}{l}\text { carriers of the T allele had lower BMI gain } \\
(\mathrm{p}<0.001)[43]\end{array}$ \\
\hline HTR2C & $\begin{array}{l}\text { rs3813929 } \\
(759 \mathrm{C} / \mathrm{T})\end{array}$ & $\begin{array}{l}\text { retrospective } \\
12 \text { months }\end{array}$ & risperidone (45) & $\begin{array}{l}\text { ASD pediatric sample; } \mathrm{n}=45 \\
(34 / 11), \mathrm{n}=31 \text { at } 12 \text { months; } \\
\text { Caucasian }(44), \text { African }(1)\end{array}$ & nonsignificant [47] \\
\hline HTR2C & $\begin{array}{l}\text { rs3813929 } \\
(759 \mathrm{C} / \mathrm{T})\end{array}$ & $\begin{array}{l}\text { cohort } \\
\geq 6 \text { months }\end{array}$ & risperidone (124) & $\begin{array}{l}\text { ASD pediatric sample (7-17 years); } \\
\mathrm{n}=124(112 / 12) ; \text { mostly European } \\
\text { American }\end{array}$ & nonsignificant [48] \\
\hline HTR2C & $\begin{array}{l}\text { rs3813929 } \\
(759 \mathrm{C} / \mathrm{T}) \\
\text { rs3813928 } \\
\text { rs498207 } \\
\text { rs6318 } \\
\text { (Ser23Cys) }\end{array}$ & $\begin{array}{l}\text { retrospective } \\
12 \text { weeks }\end{array}$ & $\begin{array}{l}\text { amisulpride }(2) \\
\text { clozapine }(24) \\
\text { olanzapine }(33) \\
\text { quetiapine }(2) \\
\text { risperidone }(8) \\
\text { mixed (52) }\end{array}$ & $\begin{array}{l}\text { SCZ or SA; } \mathrm{n}=128(80 / 48) \\
\text { European }(118), \text { Turkish }(10)\end{array}$ & $\begin{array}{l}\text { significant association for rs } 498207 \\
(\mathrm{p}<0.0049) ; \mathrm{G}-\mathrm{A}-\mathrm{T} \text { haplotype (of SNPs } \\
\text { rs498207, rs3813928, and rs3813929, } \\
\text { respectively) overrepresented in group with } \\
<7 \% \text { BWG [44] }\end{array}$ \\
\hline HTR2C & $\begin{array}{l}\text { rs3813929 } \\
(759 \mathrm{C} / \mathrm{T})\end{array}$ & $\begin{array}{l}\text { cross-sectional } \\
\geq 12 \text { months }\end{array}$ & clozapine (113) & SCZ; n = $113(81 / 32) ;$ Korean (113) & $\begin{array}{l}\text { nonsignificant trend for male weight-gainers } \\
\text { vs. male nongainers }(\mathrm{p}=0.051)[50]\end{array}$ \\
\hline HTR2C & $\begin{array}{l}\text { rs3813929 } \\
(759 \mathrm{C} / \mathrm{T})\end{array}$ & $\begin{array}{l}\text { retrospective } \\
\geq 7 \text { months }\end{array}$ & iloperidone (216) & $\begin{array}{l}\text { SCZ; } n=216(180 / 36) ; \text { White }(85) \text {, } \\
\text { Black (106), Asian (17), other (8) }\end{array}$ & nonsignificant [51] \\
\hline HTR2C & $\begin{array}{l}\text { rs3813929 } \\
(759 \mathrm{C} / \mathrm{T})\end{array}$ & $\begin{array}{l}\text { cohort } \\
\text { naturalistic } \\
95 \% \geq 3 \text { months }\end{array}$ & $\begin{array}{l}\text { aripiprazole (23) } \\
\text { clozapine }(68) \\
\text { olanzapine }(54) \\
\text { quetiapine }(31) \\
\text { risperidone }(30)\end{array}$ & $\begin{array}{l}\text { psychotic disorder (89), mood } \\
\text { disorder }(21) \text {, personality disorder } \\
(8) \text {, other }(23) ; \mathrm{n}=141(82 / 59) ; \\
\text { European-Caucasian }\end{array}$ & nonsignificant [52] \\
\hline HTR2C & $\begin{array}{l}\text { rs3813929 } \\
(759 \mathrm{C} / \mathrm{T})\end{array}$ & $\begin{array}{l}\text { clinical trial } \\
8 \text { weeks }\end{array}$ & olanzapine (205) & $\begin{array}{l}\text { bipolar disorder, borderline } \\
\text { personality disorder, treatment } \\
\text { resistant depression; } \mathrm{n}=205 \text {; White, } \\
\text { Hispanic, Black }\end{array}$ & nonsignificant [53] \\
\hline HTR2C & $\begin{array}{l}\text { rs3813929 } \\
(759 \mathrm{C} / \mathrm{T})\end{array}$ & $\begin{array}{l}\text { retrospective } \\
3 \text { months }\end{array}$ & $\begin{array}{l}\text { olanzapine or } \\
\text { risperidone }\end{array}$ & $\begin{array}{l}\text { SCZ (77), SA (7), or delusional } \\
\text { disorder (17); } \mathrm{n}=101 ; \text { drug-naïve } \\
\text { females, Caucasian (101) }\end{array}$ & nonsignificant [54] \\
\hline HTR2C & $\begin{array}{l}\text { rs3813929 } \\
(759 \mathrm{C} / \mathrm{T}) \\
\text { rs518147 } \\
(\mathrm{G} 697 \mathrm{C}) \\
\text { rs6318 } \\
\text { (Ser23Cys) }\end{array}$ & $\begin{array}{l}\text { retrospective } \\
6 \text { weeks }\end{array}$ & $\begin{array}{l}\text { mostly olanzapine } \\
\text { or clozapine }\end{array}$ & $\begin{array}{l}\text { chronic SCZ or SA; } \mathrm{n}=205(141 / 64) \text {; } \\
\text { European (126), African-American } \\
\text { (58), unspecified (21) }\end{array}$ & $\begin{array}{l}\text { C-G-Cys haplotype (for SNPs rs3813929, } \\
\text { rs518147, and rs6318, respectively) over- } \\
\text { represented in patients with weight gain } \\
(\mathrm{p}=0.0015 \text {; OR: } 1.93,95 \% \mathrm{CI}=1.04-3.56) \\
{[45]}\end{array}$ \\
\hline HTR2C & $\begin{array}{l}\text { rs3813929 } \\
(759 \mathrm{C} / \mathrm{T}) \\
\mathrm{rs} 1414334 \\
(\mathrm{C} / \mathrm{G})\end{array}$ & meta-analysis & olanzapine (336) & $\begin{array}{l}\text { chronic SCZ; } \mathrm{n}=336(140 / 196) ; \\
\text { European (149), Croatian (108), } \\
\text { Korean (79) }\end{array}$ & $\begin{array}{l}\text { nonsignificant association for }-759 \mathrm{C} / \mathrm{T} \text { allele; } \\
\text { significant association showing that C allele } \\
\text { confers risk of AP-MetS }(\mathrm{OR}: 2.44,95 \% \mathrm{CI}= \\
\left.1.48-4.00 ; \mathrm{p}=0.0004 ; \mathrm{I}^{2}=0\right)[49]\end{array}$ \\
\hline HTR2C & $\begin{array}{l}\text { rs3813929 } \\
(759 \mathrm{C} / \mathrm{T})\end{array}$ & meta-analysis & various & $\begin{array}{l}\text { SCZ or SA; } \mathrm{n}=862 ; \text { European } \\
\text { American, African-American, Asian }\end{array}$ & $\begin{array}{l}\text { T allele confers significant protection against } \\
\text { AIWG }(\mathrm{p}=0.02) \text {; association more } \\
\text { pronounced after limiting analysis to all or } \\
\text { almost all European samples }(\mathrm{p}=0.006)[45]\end{array}$ \\
\hline HTR2C & $\begin{array}{l}\text { rs1414334 } \\
(\mathrm{C} / \mathrm{G})\end{array}$ & $\begin{array}{l}\text { cross-sectional } \\
\geq 3 \text { months }\end{array}$ & $\begin{array}{l}\text { olanzapine }(43) \\
\text { risperidone }(40) \\
\text { clozapine }(31) \\
\text { aripiprazole }(11) \\
\text { quetiapine }(15) \\
\text { FGAs (17) } \\
\text { polypharmacy (29) }\end{array}$ & $\begin{array}{l}\text { SCZ (146), SA (23), or psychotic } \\
\text { disorder (17); } \mathrm{n}=186(127 / 59) ; 93 \% \\
\text { of participants were of European } \\
\text { ancestry while the remaining } 7 \% \\
\text { were of Asian, African, or mixed } \\
\text { ancestry }\end{array}$ & $\begin{array}{l}\mathrm{C} \text { allele carriership is significantly associated } \\
\text { with a greater risk of AP-MetS (OR: } 3.73,95 \% \\
\mathrm{CI}=1.29-10.79 ; \mathrm{p}=0.015)[61]\end{array}$ \\
\hline HTR2C & $\begin{array}{l}\text { rs1414334 } \\
(\mathrm{C} / \mathrm{G})\end{array}$ & $\begin{array}{l}\text { cross-sectional } \\
\text { unstated }\end{array}$ & clozapine (190) & $\begin{array}{l}\text { SCZ (clinical diagnosis of F2 group } \\
\text { according to ICD-10); } \mathrm{n}=190 ; \text { all } \\
\text { European }\end{array}$ & nonsignificant [62] \\
\hline
\end{tabular}


Table 2 (continued)

\begin{tabular}{|c|c|c|c|c|c|}
\hline Gene & $\begin{array}{l}\text { Poly- } \\
\text { morphism(s) }\end{array}$ & $\begin{array}{l}\text { Design, } \\
\text { treatment } \\
\text { duration }\end{array}$ & Medication (n) & $\begin{array}{l}\text { Sample [diagnosis; } \mathrm{n}(\mathrm{m} / \mathrm{f}) \text {; } \\
\text { ethnicity/race] }\end{array}$ & Main findings \\
\hline HTR2C & $\begin{array}{l}\text { rs6318 } \\
\text { (rs1414334 } \\
\text { proxy) } \\
\text { rs498177, } \\
\text { rs521018, } \\
\text { rs5988072, } \\
\text { rs2192371, } \\
\text { rs12833104 }\end{array}$ & $\begin{array}{l}\text { cross-sectional } \\
\geq 3 \text { months }\end{array}$ & $\begin{array}{l}\text { clozapine }(171) \\
\text { olanzapine }(91) \\
\text { risperidone }(194)\end{array}$ & SCZ; $n=456$ (299/157); Han Chinese & $\begin{array}{l}\text { the rs521018-rs } 498177 \text { haplotype was } \\
\text { significantly associated with AP-MetS, but } \\
\text { only in female patients }(\mathrm{p}=0.0108)[63]\end{array}$ \\
\hline $\begin{array}{l}\text { HTR2C- } \\
\text { LEP }\end{array}$ & $\begin{array}{l}\text { rs3813929 } \\
(759 \mathrm{C} / \mathrm{T}) \\
\text { rs7799039 } \\
(2548 \mathrm{G} / \mathrm{A})\end{array}$ & $\begin{array}{l}\text { cross-sectional } \\
\geq 3 \text { months }\end{array}$ & $\begin{array}{l}\text { clozapine }(67) \\
\text { olanzapine }(67) \\
\text { risperidone }(55) \\
\text { quetiapine }(3) \\
\text { polypharmacy }(8)\end{array}$ & $\begin{array}{l}\text { SCZ (139), SA (43), or psychotic } \\
\text { disorder }(18) ; \mathrm{n}=200(134 / 66) ; \\
\text { European-Caucasian }\end{array}$ & $\begin{array}{l}\text { combined HTR2C }-759 \mathrm{C} / \mathrm{T}-L E P-2548 \mathrm{G} / \mathrm{A} \\
\text { genotype significantly associated with AIWG } \\
\text { (OR: } 2.88 ; 95 \% \mathrm{CI}=1.05-7.95)[46]\end{array}$ \\
\hline$\overline{L E P}$ & $\begin{array}{l}\text { rs7799039 } \\
(2548 \mathrm{G} / \mathrm{A})\end{array}$ & $\begin{array}{l}\text { retrospective } \\
12 \text { weeks }\end{array}$ & $\begin{array}{l}\text { clozapine }(24) \\
\text { olanzapine }(33) \\
\text { other }(12) \\
\text { mixed }(52)\end{array}$ & $\begin{array}{l}\text { SCZ or SA; } \mathrm{n}=128(80 / 48) \\
\text { European }(118), \text { Turkish }(10)\end{array}$ & nonsignificant [44] \\
\hline$\overline{L E P}$ & $\begin{array}{l}\text { rs7799039 } \\
(2548 \mathrm{G} / \mathrm{A})\end{array}$ & $\begin{array}{l}\text { cross-sectional } \\
\geq 12 \text { months }\end{array}$ & $\begin{array}{l}\text { clozapine }(113) \\
\text { polypharmacy for } \\
39.8 \% \text { patients }\end{array}$ & SCZ; n = $113(81 / 32) ;$ Korean (113) & $\begin{array}{l}\text { BMI gain for AA genotype carriers was } \\
\text { significantly greater than carriers of both the } \\
\text { AG and GG genotypes ( } p=0.048 \text {; reported } \\
\text { for AA-AG comparison) [50] }\end{array}$ \\
\hline$\overline{L E P}$ & $\begin{array}{l}\text { rs7799039 } \\
(2548 \mathrm{G} / \mathrm{A})\end{array}$ & $\begin{array}{l}\text { cohort, } \\
\text { naturalistic } \\
95 \% \geq 3 \text { months }\end{array}$ & $\begin{array}{l}\text { aripiprazole }(23) \\
\text { clozapine }(68) \\
\text { olanzapine }(54) \\
\text { quetiapine }(31) \\
\text { risperidone }(30)\end{array}$ & $\begin{array}{l}\text { psychotic disorder (89), mood } \\
\text { disorder }(21) \text {, personality disorder } \\
\text { (8), other (23); } \mathrm{n}=141(82 / 59) ; \\
\text { European-Caucasian }\end{array}$ & nonsignificant $[52]$ \\
\hline$\overline{L E P}$ & $\begin{array}{l}\text { rs7799039 } \\
(2548 \mathrm{G} / \mathrm{A})\end{array}$ & $\begin{array}{l}\text { cross-sectional } \\
\text { unstated }\end{array}$ & clozapine (190) & $\begin{array}{l}\text { SCZ (clinical diagnosis of F2 group } \\
\text { according to ICD-10); } \mathrm{n}=190 ; \\
\text { Finnish (190) }\end{array}$ & nonsignificant [62] \\
\hline$\overline{L E P}$ & $\begin{array}{l}\text { rs7799039 } \\
(2548 \mathrm{G} / \mathrm{A})\end{array}$ & $\begin{array}{l}\text { cross-sectional } \\
\text { unstated }\end{array}$ & $\begin{array}{l}\text { clozapine }(132) \\
\text { olanzapine }(41) \\
\text { risperidone }(120) \\
\text { quetiapine }(52) \\
\text { sulpride }(48) \\
\text { haloperidol }(47)\end{array}$ & $\begin{array}{l}\text { SCZ; } \mathrm{n}=633(338 / 295) ; 570 \text { included } \\
\text { in pharmacogenetic analysis; patients } \\
\text { of Taiwanese descent }\end{array}$ & $\begin{array}{l}\text { LEP rs7799039 G allele significantly } \\
\text { associated with BMI }(\mathrm{p}=0.008) \text {, waist } \\
\text { circumference }(\mathrm{p}=0.014) \text {, MetS }(\mathrm{p}=0.019) \text {, } \\
\text { insulin }(\mathrm{p}=0.034) \text {, and HOMA }(\mathrm{p}=0.029) \\
\text { under a dominance genotypic model }[73]\end{array}$ \\
\hline$\overline{L E P}$ & $\begin{array}{l}\text { rs7799039 } \\
(2548 \mathrm{G} / \mathrm{A})\end{array}$ & $\begin{array}{l}\text { retrospective } \\
\geq 3 \text { months }\end{array}$ & clozapine (56) & $\begin{array}{l}\text { SCZ, SA, or psychotic disorder; } \mathrm{n}= \\
56(44 / 12) ; \text { Spanish }\end{array}$ & nonsignificant [76] \\
\hline$\overline{L E P}$ & $\begin{array}{l}\text { rs7799039 } \\
(2548 \mathrm{G} / \mathrm{A}) \\
\text { rs10954173 } \\
\text { rs3828942 }\end{array}$ & $\begin{array}{l}\text { prospective } \\
\geq 6 \text { weeks }\end{array}$ & $\begin{array}{l}\text { clozapine }(80) \\
\text { olanzapine }(28) \\
\text { risperidone }(32) \\
\text { haloperidol }(16) \\
\text { other }(24)\end{array}$ & $\begin{array}{l}\text { SCZ; n =181; European (127), } \\
\text { African-American (43), other (9) }\end{array}$ & $\begin{array}{l}\text { significant association between haplotype } \\
\text { LEP rs7799039G-rs10954173G-rs3828942G } \\
\text { with AIWG }(p=0.035) \text {; the rs7799039 G } \\
\text { allele }(p=0.042) \text { and G allele of rs3828942 } \\
(p=0.032) \text { were associated with greater } \\
\text { weight gain [74] }\end{array}$ \\
\hline$\overline{L E P}$ & $\begin{array}{l}\text { rs7799039 } \\
(2548 \mathrm{G} / \mathrm{A}) \\
\text { rs10244329, } \\
\text { rs12706832 } \\
\text { rs2071045 }\end{array}$ & $\begin{array}{l}\text { cohort } \\
8 \text { weeks }\end{array}$ & risperidone (181) & $\begin{array}{l}\text { ASD pediatric sample; } \mathrm{n}=181 \\
(148 / 33) ; \text { White }(125), \text { Black }(26), \\
\text { Hispanic }(10), \text { other }(16)\end{array}$ & $\begin{array}{l}\text { significant association: the rs7799039 G allele } \\
\text { acts dominantly to increase risk for AIWG } \\
\left(\mathrm{p}=1.4 \times 10^{-4}\right) \text {, while AA homozygotes are } \\
\text { relatively protected [75] }\end{array}$ \\
\hline$\overline{L E P}$ & $\begin{array}{l}\text { rs7799039 } \\
(2548 \mathrm{G} / \mathrm{A})\end{array}$ & $\begin{array}{l}\text { prospective, } \\
\text { longitudinal } \\
12 \text { months }\end{array}$ & $\begin{array}{l}\text { haloperidol } \\
\text { olanzapine } \\
\text { risperidone } \\
\text { ziprasidone } \\
\text { aripiprazole } \\
\text { quetiapine }\end{array}$ & $\begin{array}{l}\text { drug naïve, first-episode psychosis } \\
\text { with DSM-IV criteria meeting SCZ } \\
\text { or a SCZ spectrum disorder; } \mathrm{n}=205 \\
\text { (118/87); } 94 \% \text { European-Caucasian } \\
\text { (Spanish) }\end{array}$ & nonsignificant [77] \\
\hline$\overline{L E P R}$ & $\begin{array}{l}\text { rs1137101 } \\
(\mathrm{Q} 223 \mathrm{R} \\
\text { or } 668 \mathrm{~A} / \mathrm{G})\end{array}$ & $\begin{array}{l}\text { cross-sectional } \\
\geq 3 \text { months }\end{array}$ & $\begin{array}{l}\text { clozapine }(71) \\
\text { olanzapine }(68) \\
\text { risperidone }(57) \\
\text { other SGA (4) }\end{array}$ & $\begin{array}{l}\text { SCZ (139), SA (43), or other } \\
\text { psychotic disorder }(18) ; \mathrm{n}=200 \\
(134 / 66) ; \text { European-Caucasian }\end{array}$ & $\begin{array}{l}\text { significant association for female subjects: } \\
70.6 \% \text { were obese in the } L E P R 223 \mathrm{QQ} \text { group } \\
\text { vs. } 38.5 \% \text { in the } 223 \mathrm{QR}(\mathrm{OR}: 0.11,95 \% \mathrm{CI}= \\
0.02-0.54 ; \mathrm{p}=0.007) \text { and } 40.0 \% \text { in the } 223 \mathrm{RR} \\
\text { (OR: } 0.07,95 \% \mathrm{CI}=0.01-0.63 ; \mathrm{p}=0.018) \\
\text { groups [78] }\end{array}$ \\
\hline
\end{tabular}

Genetics of Common AntipsychoticInduced Adverse Effects
Mol Neuropsychiatry 2016;2:61-78 DOI: $10.1159 / 000445802$ 
Table 2 (continued)

\begin{tabular}{|c|c|c|c|c|c|}
\hline Gene & $\begin{array}{l}\text { Poly- } \\
\text { morphism(s) }\end{array}$ & $\begin{array}{l}\text { Design, } \\
\text { treatment } \\
\text { duration }\end{array}$ & Medication (n) & $\begin{array}{l}\text { Sample [diagnosis; } \mathrm{n}(\mathrm{m} / \mathrm{f}) \\
\text { ethnicity/race] }\end{array}$ & Main findings \\
\hline$L E P R$ & $\begin{array}{l}\text { rs1137101 } \\
(\mathrm{Q} 223 \mathrm{R} \\
\text { or } 668 \mathrm{~A} / \mathrm{G})\end{array}$ & $\begin{array}{l}\text { cohort, } \\
\text { naturalistic } \\
95 \% \geq 3 \text { months }\end{array}$ & $\begin{array}{l}\text { aripiprazole }(23) \\
\text { clozapine }(68) \\
\text { olanzapine }(54) \\
\text { quetiapine }(31) \\
\text { risperidone }(30)\end{array}$ & $\begin{array}{l}\text { psychotic disorder }(89), \text { mood } \\
\text { disorder }(21) \text {, personality disorder } \\
\text { (8), other }(23) ; \mathrm{n}=141(82 / 59) ; \\
\text { European-Caucasian }\end{array}$ & $\begin{array}{l}\text { LEPR } 223 \mathrm{R} \text { allele was significantly associated } \\
\text { with an increased risk of obesity in females } \\
(\mathrm{p}=0.03) \text {, but not in males [52] }\end{array}$ \\
\hline$\overline{L E P R}$ & $\begin{array}{l}\text { rs1137101 } \\
(\mathrm{Q} 223 \mathrm{R} \\
\text { or 668A/G) }\end{array}$ & $\begin{array}{l}\text { cross-sectional } \\
\text { unstated }\end{array}$ & $\begin{array}{l}\text { clozapine }(132) \\
\text { olanzapine }(41) \\
\text { risperidone }(120) \\
\text { other (147) }\end{array}$ & $\begin{array}{l}\text { SCZ; } \mathrm{n}=633(338 / 295) ; 570 \text { included } \\
\text { in pharmacogenetic analysis; patients } \\
\text { of Taiwanese descent }\end{array}$ & nonsignificant [73] \\
\hline$\overline{L E P R}$ & $\begin{array}{l}\text { rs1137101 } \\
(\mathrm{Q} 223 \mathrm{R} \\
\text { or } 668 \mathrm{~A} / \mathrm{G})\end{array}$ & $\begin{array}{l}\text { retrospective } \\
\geq 6 \text { weeks }\end{array}$ & $\begin{array}{l}\text { clozapine }(80) \\
\text { olanzapine }(28) \\
\text { risperidone }(32) \\
\text { haloperidol }(16) \\
\text { other }(24)\end{array}$ & $\begin{array}{l}\text { SCZ; } n=181, \text { European (127) } \\
\text { African-American (43), other (9) }\end{array}$ & nonsignificant [74] \\
\hline$\overline{L E P R}$ & $\begin{array}{l}\text { rs1137101 } \\
(\mathrm{Q} 223 \mathrm{R} \\
\text { or } 668 \mathrm{~A} / \mathrm{G})\end{array}$ & $\begin{array}{l}\text { prospective, } \\
\text { longitudinal } \\
12 \text { months }\end{array}$ & $\begin{array}{l}\text { haloperidol } \\
\text { olanzapine } \\
\text { risperidone } \\
\text { quetiapine }\end{array}$ & $\begin{array}{l}\text { drug naïve, first-episode psychosis } \\
\text { with DSM-IV criteria meeting SCZ } \\
\text { or a SCZ spectrum disorder; } \mathrm{n}=205 \\
\text { (118/87); } 94 \% \text { European-Caucasian } \\
\text { (Spanish) }\end{array}$ & nonsignificant [77] \\
\hline$\overline{L E P R}$ & $\begin{array}{l}\text { rs1137101 } \\
(\mathrm{Q} 223 \mathrm{R} \\
\text { or } 668 \mathrm{~A} / \mathrm{G})\end{array}$ & $\begin{array}{l}\text { cross-sectional } \\
\geq 12 \text { months }\end{array}$ & $\begin{array}{l}\text { olanzapine (62) } \\
\text { risperidone (59) } \\
\text { paliperidone (24) } \\
\text { other APs (61) }\end{array}$ & $\begin{array}{l}\mathrm{n}=206(137 / 69) ; \text { Malaysian (109), } \\
\text { Chinese (76), Indian (21) }\end{array}$ & $\begin{array}{l}\text { LEPR 223R or 668G allele significantly } \\
\text { associated with increased risk of MetS (OR: } \\
0.47,95 \% \mathrm{CI}=0.28-0.80, \mathrm{p}=0.005)[81]\end{array}$ \\
\hline$\overline{M C 4 R}$ & $\begin{array}{l}\text { rs17782313 } \\
(\mathrm{C} / \mathrm{T}) \\
\text { rs2229616 } \\
\text { rs11872992 } \\
\text { rs8087522 }\end{array}$ & $\begin{array}{l}\text { cohort } \\
4-14 \text { weeks }\end{array}$ & $\begin{array}{l}\text { clozapine (99) } \\
\text { olanzapine (36) } \\
\text { risperidone }(40) \\
\text { haloperidol (16) } \\
\text { other (33) }\end{array}$ & $\begin{array}{l}\text { chronic SCZ or SA; } \mathrm{n}=224(150 / 74) \\
\text { European American }(157) \text {, African- } \\
\text { American (56), other (11) }\end{array}$ & $\begin{array}{l}\text { nonsignificant trend with respect to } \\
\text { rs } 17782313 \text { ( } \mathrm{p}=0.09) \text {; nominally significant } \\
\text { association for rs } 8087522 \text { detected in the } \\
\text { clozapine-treated European subsample, with } \\
\text { the A allele being overrepresented within the } \\
\text { weight gain group [97] }\end{array}$ \\
\hline$M C 4 R$ & $\begin{array}{l}\text { rs17782313 } \\
(\mathrm{C} / \mathrm{T})\end{array}$ & $\begin{array}{l}\text { retrospective } \\
4 \text { weeks }\end{array}$ & $\begin{array}{l}\text { olanzapine (135) } \\
\text { quetiapine }(102) \\
\text { risperidone }(66) \\
\text { amisulpride }(58) \\
\text { clozapine (36) } \\
\text { paliperidone }(28)\end{array}$ & $\begin{array}{l}\text { SCZ; whole study, } \mathrm{n}=345,61 \% \text { with } \\
\text { an F2 (ICD-10) diagnosis; } \\
\text { adjusted subsample (controlling for } \\
\text { co-medication), } \mathrm{n}=173,80 \% \text { with } \mathrm{F} 2 \\
\text { diagnosis; first-episode sample, } \mathrm{n}= \\
\text { 59; control sample, } \mathrm{n}=40\end{array}$ & $\begin{array}{l}\mathrm{C} \text { allele was significantly associated with risk } \\
\text { of weight gain in a dose-loading manner }(\mathrm{p}= \\
0.045) \text {; effect was more pronounced in the } \\
\text { adjusted subsample excluding co-medication } \\
(\mathrm{p}=0.03) \text {; dose-response effect was absent in } \\
\text { first-episode subsample, but association was } \\
\text { still significant }(\mathrm{p}=0.041)[98]\end{array}$ \\
\hline$\overline{M C 4 R}$ & $\begin{array}{l}\text { rs8087522 } \\
\text { rs489693 } \\
(\mathrm{A} / \mathrm{C}) \\
\text { rs11872992 } \\
\text { rs8093815 }\end{array}$ & $\begin{array}{l}\text { cohort } \\
8 \text { weeks }\end{array}$ & risperidone (181) & $\begin{array}{l}\text { ASD pediatric sample; } n=181 \\
(148 / 33) ; \text { White }(125), \text { Black }(26), \\
\text { Hispanic }(10), \text { other }(16)\end{array}$ & $\begin{array}{l}\text { nonsignificant trends for associations of } \\
\text { rs } 8087522 \text { and } \mathrm{rs} 8093815 \text { with AIWG ( } \mathrm{p}= \\
0.06 \text { and } \mathrm{p}=0.07 \text {, respectively); nominally } \\
\text { significant results for associations of } \\
\text { rs } 11872992 \text { and } \mathrm{rs} 489693 \text { with AIWG ( } \mathrm{p}= \\
\left.0.03 \text { for both; } \mathrm{p}_{\mathrm{cor}}>0.05\right)[75]\end{array}$ \\
\hline$\overline{M C 4 R}$ & Tag-SNPs & $\begin{array}{l}\text { GWAS, cohort } \\
6-12 \text { weeks }\end{array}$ & $\begin{array}{l}\text { cohort 1: } \\
\text { quetiapine (36) } \\
\text { risperidone }(135) \\
\text { aripiprazole }(41) \\
\text { olanzapine }(45) \\
\text { cohort 2: clozapine } \\
(73) \\
\text { cohort 3: various }\end{array}$ & $\begin{array}{l}\text { cohort 1: drug-naïve youths (4-19 } \\
\text { years), } \mathrm{n}=270 \text {; European American } \\
(131), \text { African-American (70), other } \\
(69) \\
\text { cohort 2: treatment-resistant SCZ, } \\
\mathrm{n}=73 \text {; all European American } \\
\text { cohort 3: SCZ, } \mathrm{n}=40\end{array}$ & $\begin{array}{l}\text { rs } 489693 \text { A allele showed significant effects in } \\
\text { all three cohorts: } \\
\text { cohort } 1: \mathrm{p}=2.8 \times 10^{-7} \\
\text { cohort } 2: \mathrm{p}=1.4 \times 10^{-4} \\
\text { cohort } 3: \mathrm{p}=0.007 \\
\text { meta-analytic } \mathrm{p}=5.59 \times 10^{-12}[93]\end{array}$ \\
\hline$\overline{M C 4 R}$ & rs489693 & $\begin{array}{l}\text { retrospective } \\
4 \text { weeks }\end{array}$ & $\begin{array}{l}\text { olanzapine }(133) \\
\text { quetiapine }(102) \\
\text { risperidone }(66) \\
\text { clozapine }(34) \\
\text { other }(83)\end{array}$ & $\begin{array}{l}\text { whole study, } \mathrm{n}=341, \sim 61 \% \text { with an } \\
\text { F2 (ICD-10) diagnosis; adjusted } \\
\text { subsample, } \mathrm{n}=169, \sim 80 \% \text { with } \mathrm{F} 2 \\
\text { diagnosis; first-episode sample, } \mathrm{n}= \\
\text { 59; control sample, } \mathrm{n}=40\end{array}$ & $\begin{array}{l}\text { MC4R rs489693 A allele was significantly } \\
\text { associated with increased risk of AIWG in } \\
\text { whole study sample and both of the } \\
\text { subsamples [96] }\end{array}$ \\
\hline MTHFR & $\begin{array}{l}\text { rs1801133 } \\
(\mathrm{C} 677 \mathrm{~T})\end{array}$ & $\begin{array}{l}\text { cross-sectional } \\
\text { median } \\
\text { treatment } \\
\text { duration of } 6 \\
\text { months }\end{array}$ & $\begin{array}{l}\text { quetiapine (49) } \\
\text { risperidone }(46) \\
\text { other }(10)\end{array}$ & $\begin{array}{l}\text { SGA-treated sample, } \mathrm{n}=105 \\
\text { primarily of European descent }(74 \%)\end{array}$ & $\begin{array}{l}\mathrm{T} \text { allele carriership was significantly } \\
\text { associated with a greater prevalence of MetS } \\
(\mathrm{p}=0.05)[108]\end{array}$ \\
\hline
\end{tabular}


Table 2 (continued)

\begin{tabular}{|c|c|c|c|c|c|}
\hline Gene & $\begin{array}{l}\text { Poly- } \\
\text { morphism(s) }\end{array}$ & $\begin{array}{l}\text { Design, } \\
\text { treatment } \\
\text { duration }\end{array}$ & Medication (n) & $\begin{array}{l}\text { Sample [diagnosis; } \mathrm{n}(\mathrm{m} / \mathrm{f}) \text {; } \\
\text { ethnicity/race] }\end{array}$ & Main findings \\
\hline MTHFR & $\begin{array}{l}\text { rs1801131 } \\
(\mathrm{A} 1298 \mathrm{C}) \\
\text { rs1801133 } \\
(\mathrm{C} 677 \mathrm{~T})\end{array}$ & $\begin{array}{l}\text { cohort } \\
8.87 \pm 3.6 \\
\text { weeks }\end{array}$ & $\begin{array}{l}\text { clozapine or } \\
\text { olanzapine (179) } \\
\text { risperidone (92) } \\
\text { other }(77)\end{array}$ & $\begin{array}{l}\mathrm{n}=347(183 / 164) ; 66 \text { were first- } \\
\text { episode patients; European/ } \\
\text { Caucasian (281), African-Americans } \\
\text { (56), other (11) }\end{array}$ & $\begin{array}{l}\text { nonsignificant association for MTHFR } \\
\text { A1298C variant in total study sample, } \\
\text { clozapine/olanzapine-treated European } \\
\text { subsample, and first-episode subsample } \\
\text { analyses; nominal association detected for the } \\
\text { C677T variant in total study sample analysis } \\
\left(\mathrm{p}=0.03 ; \mathrm{p}_{\text {cor }}>0.05\right)[109]\end{array}$ \\
\hline MTHFR & $\begin{array}{l}\text { rs1801131 } \\
(\mathrm{A} 1298 \mathrm{C}) \\
\text { rs1801133 } \\
(\mathrm{C} 677 \mathrm{~T})\end{array}$ & $\begin{array}{l}\text { cohort } \\
\text { Chinese } \\
\text { sample: } 8-10 \\
\text { weeks } \\
\text { Spanish sample: } \\
3 \text { months } \\
\end{array}$ & $\begin{array}{l}\text { Chinese sample: } \\
\text { chlorpromazine } \\
\text { risperidone, other } \\
\text { Spanish sample: } \\
\text { risperidone } \\
\text { olanzapine, other }\end{array}$ & $\begin{array}{l}\text { first-episode, drug-naïve SCZ } \\
\text { patients; Chinese Han sample, } \mathrm{n}= \\
182(83 / 99) ; \text { Spanish sample, } \mathrm{n}=72 \\
(53 / 19)\end{array}$ & $\begin{array}{l}\text { 677CC genotype carriers showed a } \\
\text { significantly greater gain in BMI compared to } \\
\text { T allele carriers for both the Chinese }(\mathrm{p}= \\
0.012) \text { and Spanish }(\mathrm{p}=0.017) \text { samples; } \\
\text { findings for the rs1801131 SNP were } \\
\text { nonsignificant [110] }\end{array}$ \\
\hline$\overline{M T H F R}$ & $\begin{array}{l}\text { rs1801131 } \\
(\mathrm{A} 1298 \mathrm{C}) \\
\text { rs1801133 } \\
(\mathrm{C} 677 \mathrm{~T})\end{array}$ & $\begin{array}{l}\text { cross-sectional } \\
\text { variable }\end{array}$ & primarily SGAs & $\begin{array}{l}\text { SCZ }(69.3 \%), \text { SD }(11.4 \%) \text {, or SA } \\
(19.3 \%) ; \mathrm{n}=518(66.4 \% \text { male }) ; \\
\text { primarily of European ancestry }\end{array}$ & $\begin{array}{l}\text { significant association detected for } M T H F R \\
\text { A1298C, with C/C genotype carriers having a } \\
2.4 \text { times higher risk of MetS compared to } \\
\text { A/A genotypes (OR: } 2.44,95 \% \mathrm{CI}=1.25- \\
\text { 4.76; } \mathrm{p}=0.009 \text { ) [111] }\end{array}$ \\
\hline$\overline{M T H F R}$ & $\begin{array}{l}\text { rs1801131 } \\
(\mathrm{A} 1298 \mathrm{C}) \\
\text { rs1801133 } \\
(\mathrm{C} 677 \mathrm{~T})\end{array}$ & $\begin{array}{l}\text { cohort } \\
3 \text { months }\end{array}$ & $\begin{array}{l}\text { clozapine (16) } \\
\text { olanzapine (41) } \\
\text { risperidone (37) } \\
\text { other (30) }\end{array}$ & $\begin{array}{l}\text { SCZ or SA; } \mathrm{n}=104(71 / 33) ; \\
\text { European-Caucasian }\end{array}$ & $\begin{array}{l}\text { MTHFR } 1298 \mathrm{C} \text { allele was significantly } \\
\text { associated with changes to several metabolic } \\
\text { parameters after SGA initiation, especially } \\
\text { weight gain }(\mathrm{p}=0.006) \text { and changes in blood } \\
\text { glucose }(\mathrm{p}=0.024)[112]\end{array}$ \\
\hline MTHFR & $\begin{array}{l}\text { rs1801133 } \\
\text { (C677T) }\end{array}$ & $\begin{array}{l}\text { cross-sectional } \\
\geq 12 \text { months }\end{array}$ & $\begin{array}{l}\text { olanzapine (62) } \\
\text { risperidone (59) } \\
\text { paliperidone }(24) \\
\text { other APs }(61)\end{array}$ & $\begin{array}{l}\text { SCZ; } \mathrm{n}=206(137 / 69) ; \text { Malaysian } \\
(109), \text { Chinese (76), Indian (21) }\end{array}$ & $\begin{array}{l}\text { nominally significant: } M T H F R 677 \mathrm{~T} \text { allele } \\
\text { appeared to confer protection against AP- } \\
\text { MetS (OR: } 0.59,95 \% \mathrm{CI}=0.35-0.99, \mathrm{p}= \\
0.049 \text { ); authors declare that correction for } \\
\text { multiple testing was not performed to avoid } \\
\text { type } 2 \text { error [81] }\end{array}$ \\
\hline MTHFR & $\begin{array}{l}\text { rs1801133 } \\
(\mathrm{C} 677 \mathrm{~T})\end{array}$ & $\begin{array}{l}\text { cross-sectional } \\
\geq 6 \text { months }\end{array}$ & various & $\begin{array}{l}\text { SCZ spectrum disorder; } \mathrm{n}=127 \text {; } \\
\text { ethnicity unspecified }\end{array}$ & $\begin{array}{l}\text { significant association: for patients using risk } \\
\text { medication, T allele carriership (CT/TT) was } \\
\text { associated with an increased risk of AP-MetS } \\
(\mathrm{p}<0.001) ; M T H F R \text { and COMT } 158 \mathrm{Val} \\
\text { interaction was significant }(\mathrm{p}=0.0073)[107]\end{array}$ \\
\hline OGFRL1 & Tag-SNPs & $\begin{array}{l}\text { GWAS, cohort } \\
\text { discovery } \\
\text { sample: } 20.5 \\
( \pm 6.2) \\
\text { replication } \\
\text { sample: } 6.1 \\
( \pm 2.1)\end{array}$ & $\begin{array}{l}\text { discovery: } \\
\text { olanzapine (63) } \\
\text { quetiapine }(67) \\
\text { risperidone (59) } \\
\text { replication: } \\
\text { clozapine (69) } \\
\text { olanzapine (18) }\end{array}$ & $\begin{array}{l}\text { discovery sample, SCZ, } \mathrm{n}=189 \\
(151 / 38) ; \text { a subset of patients from } \\
\text { the CATIE sample of sole European } \\
\text { ancestry; replication sample, SCZ, } \\
\mathrm{n}=86(56 / 30) ; \text { all of European } \\
\text { ancestry }\end{array}$ & $\begin{array}{l}\text { GWAS yielded a number of signals in } \\
\text { association with BMI gain, the strongest of } \\
\text { which emerged from the rs } 9346455 \text { SNP } \\
\text { upstream of the OGFRL1 gene } \\
\left(\mathrm{p}=6.49 \times 10^{-6}\right) \text {; replication: } \mathrm{p}=0.005 \text {; } \\
\text { meta-analytic } \mathrm{p}=1.09 \times 10^{-7}[115]\end{array}$ \\
\hline
\end{tabular}

$\mathrm{SA}=$ Schizoaffective disorder; $\mathrm{SD}=$ schizophreniform disorder.

cant heterogeneity and publication bias that was initially detected.

A more recent meta-analysis only detected a trending association between $-759 \mathrm{C} / \mathrm{T}$ and AIWG, though only 4 studies were included as the authors were specifically interested in olanzapine-induced weight gain [49]. Kang et al. [50] also reported a trend in their study involving a sample of Korean SCZ patients. Five other studies reported neither an association nor a trend [51-55]. As Wallace et al. [41] first pointed out, contradictory reports relating to this association can mainly be attributed to differences in study duration, as the relationship between AIWG and $-759 \mathrm{C} / \mathrm{T}$ is most prominent during the early stages of treatment ( $<3$ months). Indeed, this pattern is largely consistent with the studies reviewed here. Conflicting results may also be attributable to as yet unidentified confounding variables, such as differential RNA editing [56, 57] and the 'flip-flop' phenomenon described by Lin et al. [58]. All things considered, the overall evidence suggests that the HTR2C $-759 \mathrm{C} / \mathrm{T}$ polymorphism is an important 
mediator of AIWG during the initial stages of treatment. However, further investigation is needed to determine the precise nature of this association, and also to understand how $-759 \mathrm{C} / \mathrm{T}$ may interact with other variables to influence AIWG.

Recent evidence has also reaffirmed an association of the HTR2C intragenic rs1414334 (C>G) SNP with a heightened risk of AP-MetS. Carriers of the $\mathrm{C}$ allele are more likely to meet criteria for AP-MetS. In agreement with their previous findings $[59,60]$, a Dutch research group reported an association (when controlling for type 2 error) between the rs1414334 $\mathrm{C}$ allele and a higher risk of developing AP-MetS [61]. A recent meta-analysis by $\mathrm{Ma}$ et al. [49] was able to corroborate the significance of this association when examining all 3 studies conducted by the Dutch research group. However, negative results of this association have also been reported [62, 63]. Nevertheless, there is an overall greater amount of evidence supporting this association than there is refuting it. Future studies should investigate the clinical utility of this variant as a means to inform and optimize treatment selection.

\section{$L E P$ and $L E P R$}

Leptin plays a critical role in the regulation of energy homeostasis and feeding behavior. In the hypothalamus, particularly the arcuate and ventromedial nuclei, leptin targets leptin receptors encoded by the $L E P R$ gene, whereby it transmits a potent anorectic signal [64-66]. Mutations in both $L E P$ and $L E P R$ are linked to metabolic abnormalities and the occurrence of human obesity [6769]. The LEP -2548G allele, for instance, has been associated with an increased risk of overweight and obesity [69-71]. Taken together, there is a sound theoretical basis supporting the potential involvement of $L E P$ and $L E P R$ in AP-induced metabolic dysregulation. Indeed, several studies from our review have implicated these genes in metabolic AAEs [46, 52, 72-75]. Still, negative and contradictory results have also been reported $[44,62$, $76,77]$.

In line with a 2008 report by Yevtushenko et al. [72], Gregoor et al. [46] found that the combined presence of the LEP $-2548 \mathrm{G}$ allele and absence of the HTR2C $-759 \mathrm{~T}$ allele was associated with a greater risk of SGA-related obesity. Another study by Gregoor et al. [52] found that baseline obesity risk was significantly greater for females carrying the $L E P R$ 223R allele. This result runs contrary to the finding from their 2009 study which instead reported that the 223R allele was associated with lower baseline risk for female obesity [78].
Nurmi et al. [75] have offered compelling evidence in support of the association of the $-2548 \mathrm{G}$ allele with AIWG. The sample of this study was comprised of risperidone-treated autistic youth who had participated in one of two clinical trials conducted by the NIMH Research Units on Pediatric Psychopharmacology (RUPP). The majority of subjects were drug-naïve and of European ancestry. Genotype analysis revealed a robust result for the LEP-2548G allele, which acted dominantly to confer risk of AIWG. Highly significant findings were also identified for the CNR1 promoter SNP rs806378 and the CNR1 variant rs1049353. Under a risk-allele dose model, the combination of risk variants associated with $L E P$ and CNR1 attained an impressive effect size $(D=0.85 ; \mathrm{p}=1.3 \times$ $10^{-9}$ ) [75]. Furthermore, the CNR1 rs806378 finding is in agreement with a previous report from our group [79].

Perez-Iglesias et al. [77] found no association for either the -2548G/A or Q223R SNP with AIWG. Negative findings for associations of $-2548 \mathrm{G} / \mathrm{A}$ or Q223R with AIWG were also reported by 3 other studies $[44,62,76]$. In terms of other metabolic abnormalities, Gregoor et al. [80] found that the absence of the $L E P-2548 \mathrm{G}$ and $L E P R 223 \mathrm{R}$ alleles were independently associated with higher TC/ HDL ratios in males and females, respectively. Roffeei et al. [81] reported that the $L E P R 223 \mathrm{R}$ allele was protective against AP-MetS [81]. In summary, the results for the LEP-2548G/A variant have been somewhat consistent, whereas those for the LEPR Q223R SNP are less straightforward.

\section{$M C 4 R$}

The gene product of $M C 4 R$, the melanocortin 4 receptor (MC4-R), is essential for maintaining energy homeostasis and regulating food consumption [82]. MC4-R has also been implicated in molecular pathways regulating sexual arousal, inflammatory response, pain modulation, and blood pressure [83-86]. MC4-R is widely distributed throughout the brain, though it primarily regulates metabolic pathways from within hypothalamic and brain stem nuclei $[82,87]$. MCR-4 interacts with both the serotonergic and leptinergic systems $[88,89]$, and similar to $L E P$ and $L E P R$, mutations in the MC4R gene or adjacent regions have a well-established role in the expression of congenital and polygenic forms of obesity [90-92]. Recent pharmacogenetic studies have identified the MC4-R gene locus SNPs rs489693 and rs17782313 as potential biomarkers of AIWG.

The rs489693 SNP was first detected in a genome-wide association study (GWAS) conducted by Malhotra et al. [93] in a cohort of SGA-treated, drug-naïve youth. Twen-
68

Mol Neuropsychiatry 2016;2:61-78 DOI: $10.1159 / 000445802$
MacNeil/Müller 
ty SNPs surpassed a statistical threshold of $\mathrm{p}<10^{-5}$, all of them located at a single locus, approximately $190 \mathrm{~kb}$ downstream of $M C 4 R$. This locus overlaps with a region that had been previously linked to obesity and BMI by other GWA studies [94, 95]. The association of SNP rs489693 with AIWG was subsequently confirmed in three replication cohorts. Importantly, these cohorts consisted of adult SCZ patients, and one of these cohorts had previous SGA exposure, thereby demonstrating that this association is neither exclusive to pediatric nor drug naïve populations [93]. Furthermore, this association was recently replicated by Czerwensky et al. [96] in a naturalistic study involving a sample of SCZ inpatients. A trending association between rs489693 and BMI gain was also reported by Nurmi et al. [75] in the RUPP study mentioned above.

In a study involving a sample of SCZ patients of primarily European or African ancestry (70 and 25\%, respectively), Chowdhury et al. [97] were unable to identify an association between MC4R rs17782313 and AIWG at the genotype level. However, an allelic analysis restricted to patients of European ancestry receiving either clozapine or olanzapine revealed a trending association in which the $\mathrm{C}$ allele conferred a greater risk of AIWG. Czerwensky et al. [98] were able to corroborate this finding at the genotype level and showed that CC and CT genotype carriers were at a significantly greater risk of AIWG. A haplotype analysis of rs17782313 and rs489693, as well as other SNPs in LD, may yield more robust results. In sum, the $M C 4 R$ locus appears to be a very promising candidate for the prediction of AIWG. Clinical trials will be required to assess the clinical utility of these SNP as biomarkers to predict AIWG.

\section{MTHFR}

MTHFR (methylenetetrahydrofolate reductase) encodes an enzyme that catalyzes biochemical reactions important to the folate pathway [99]. Two common MTHFR variants, rs1801133 (C677T) and rs1801131 (A1298C), reduce the catalytic activity of MTHFR by 35 and 20\%, respectively [100-102]. The attenuation of MTHFR as a consequence of these SNPs has been linked to elevated plasma homocysteine. Hyperhomocysteinemia has been associated with a greater risk of both SCZ and cardiovascular disease [103-105]. Taken together, there is a rationale establishing MTHFR as a potential candidate influencing AAEs.

Following up on the results of a previous study [106], Ellingrod et al. [107] found that an interaction between the MTHFR $677 \mathrm{~T}$ and COMT $158 \mathrm{Val}$ alleles was posi- tively associated with risk of AP-MetS. Interestingly, increasing age was negatively correlated with the likelihood of meeting MetS criteria, suggesting that this interaction exerts a stronger effect on relatively younger patients. Consistent with the studies by Ellingrod et al. [106, 107], Devlin et al. [108] found that pediatric patients with ASDs carrying the $677 \mathrm{~T}$ allele were at a greater risk of MetS compared to CC homozygotes. Similarly, Kao et al. [109] reported a nominally significant association between the $\mathrm{T}$ allele and AIWG. Conflicting results in which the $\mathrm{T}$ allele has a protective effect against AP-MetS have also been reported $[81,110]$. In addition, other studies have failed to identify an association between MetS and the 677T allele, but have reported positive results for an association with the $1298 \mathrm{C}$ allele $[111,112]$.

In light of the discordant findings, it is unlikely that MTHFR plays a major role in the PGx of metabolic AAEs. However, considering the heterogeneity across studies and possible gene interaction effects, it would be premature to completely rule out MTHFR as a potentially informative biomarker. There may also be other confounding variables not taken into consideration. For example, dietary folate has been shown to be important in moderating the effects of the MTHFR SNPs C677T and A1298C in other contexts (e.g., susceptibility to cancer) and may similarly influence AAEs [113].

\section{OGFRL1}

OGFRL1 encodes for opioid growth factor receptorlike 1, a paralog receptor which binds the endogenous opioid met-enkephalin [114]. Our research team recently conducted a GWAS to assess the genetic factors contributing to AIWG within a subset of European participants $(\mathrm{n}=189)$ derived from the Clinical Antipsychotic Trials of Intervention Effectiveness (CATIE) sample [11, 115]. This subsample represented a relatively homogenous group of participants with similar clinical characteristics and treatment. A strong signal was detected at marker rs9346455, approximately $6.6 \mathrm{~kb}$ upstream of OGFRL1, with greater BMI gain observed among carriers of the $G$ allele. This finding was subsequently replicated in an independent sample to produce a meta-analytic $p$ value of $1.09 \times 10^{-7}$. Although not quite reaching genome-wide significance, this finding still represents a robust and promising result. At present, the rs9346455 SNP is not known to have any functional significance, and only a few studies on OGFLR1 have been published [115]. Because it is unknown if or how opioid growth factor receptor-like 1 is related to energy homeostasis, this result should be interpreted with caution. Future research should aim to 
Table 3. Putative genetic associations with antipsychotic-induced TD and EPS studied from 2010 to 2015 , and their related listings by expert PGx panels

\begin{tabular}{|c|c|c|c|c|}
\hline Gene & Polymorphism & Putative association & Listings by expert panels & References \\
\hline CYP2D6 & $\begin{array}{l}\text { CYP2D } 6 * 1, * 2, * 3 \\
* 4, * 5, * 6, * 10, * 41\end{array}$ & $\begin{array}{l}\text { PM and IM status associated with a greater risk } \\
\text { of TD or EPS }\end{array}$ & PharmGKB: evidence level $3^{\text {a }}$ & {$[124-131]$} \\
\hline DPP6 & rs6977820 $(\mathrm{T}>\mathrm{C})$ & $\mathrm{T}$ allele associated with a greater risk of TD & PharmGKB: evidence level 2B & [149] \\
\hline DRD2 & rs1800497 (Taq1A) & $\mathrm{G}$ allele associated with a greater risk of TD & $\begin{array}{l}\text { PharmGKB: evidence level 2Bb } \\
\text { CPIC: evidence level } \mathrm{D}^{\mathrm{c}}\end{array}$ & {$[136-139]$} \\
\hline HSPG2 & $\begin{array}{l}\text { rs2445142 }(\mathrm{G}>\mathrm{C}) \\
\text { rs878949 }(\mathrm{T}>\mathrm{C})^{\mathrm{d}}\end{array}$ & $\begin{array}{l}\mathrm{G} \text { and } \mathrm{T} \text { allele associated with a greater risk } \\
\text { of TD }\end{array}$ & PharmGKB: evidence level $3^{a}$ & {$[145-147]$} \\
\hline
\end{tabular}

References include both positive and negative findings. See references $[19,20]$ for further information on the PharmGKB and CPIC, as well as the criteria used to assign evidence levels. ${ }^{a}$ Low evidence for this association. ${ }^{b}$ Moderate evidence for this association. ${ }^{\mathrm{c}}$ Considered of interest. ${ }^{\mathrm{d}}$ Perfect proxy marker for $\mathrm{rs} 2445142\left(\mathrm{r}^{2}=1\right)$.

address these uncertainties and clarify the potential role of OGFRL1 in AIWG.

To summarize this section, several genetic associations have been consistently replicated for AIWG, and the HTR2C intragenic SNP rs1414334 has been verified as a possible predictor of MetS vulnerability. In light of this accumulating evidence, efforts have been undertaken to develop the first gene panels for use in clinical populations [e.g., 116].

\section{EPS and TD}

Our review of EPS and TD will focus on the genes encoding cytochrome P450 2D6 (CYP2D6), dopamine receptor D2 (DRD2), heparan sulfate proteoglycan 2 (HPSG2), dipeptidyl aminopeptidase-like protein 6 (DPP6), and vesicular monoamine transporter 2 (SLC18A2) genes. See table 3 for an overview of the results and listings by expert PGx panels. Detailed information on the studies is listed in table 4 .

\section{CYP2D6}

CYP2D6 is a highly polymorphic enzyme involved in the metabolism of over $25 \%$ of the pharmaceuticals in clinical use, including the majority of APs [24]. CYP2D6 is predominantly expressed within the liver [117], though constitutive expression has also been detected in various brain regions, suggesting that CYP2D6 may also influence the activity of drugs at their sites of action [118].
Given the highly polymorphic nature of the CYP2D6 locus, genotypes are typically described using *star allele nomenclature, which indicates an estimate of an individual's corresponding phenotype or metabolizer status [119]. Four different metabolizer phenotypes are commonly identified: (1) poor metabolizer (PM); (2) intermediate metabolizer (IM); (3) extensive metabolizer (EM; the 'wild-type'), and (4) ultrarapid metabolizer. The frequencies of these phenotypes and corresponding genotypes vary considerably between ethnic groups. For additional details on the specific allelic combinations associated with each metabolizer status and the distribution of CYP2D6 alleles among different ethnic groups, refer to Hicks et al. [21] and references $[17,120]$, respectively. Numerous studies published prior to 2010 have examined the relationship between CYP2D6 and EPS/TD, with the majority having supported a significant association between CYP2D6 metabolizer status and susceptibility to EPS/TD [121-123]. Eight studies published since 2010 have been reviewed here [124-131].

Fleeman et al. [124] conducted a meta-analysis of 20 studies reporting data on EPS and/or TD in relation to the CYP2D6 genotype. The majority of studies included clinical samples of patients treated with FGAs. After limiting the analysis to prospective studies only, PMs and IMs were found to have a significantly greater susceptibility to developing TD and AP-induced parkinsonism than EMs. Additionally, PMs had TD symptoms of greater severity than EMs. 
Table 4. Overview of studies pertaining to antipsychotic-induced movement disorders

\begin{tabular}{|c|c|c|c|c|c|}
\hline Gene & Polymorphism(s) & $\begin{array}{l}\text { Design, treatment } \\
\text { duration }\end{array}$ & Medication (n) & Sample [diagnosis; n (m/f); ethnicity] & Main findings \\
\hline CYP2D6 & EM/UM, IM, PM & meta-analysis & primarily FGAs & $\begin{array}{l}\text { SCZ spectrum disorder; association } \\
\text { of TD with CYP2D6, } \mathrm{n}=1,482 \text { (all } \\
\text { studies); } \mathrm{n}=336 \text { (prospective); } \\
\text { Caucasian, Japanese, Korean, Chinese }\end{array}$ & $\begin{array}{l}\text { analysis of prospective studies showed } \\
\text { significant associations between TD and } \\
\text { CYP2D6 when comparing wt/wt genotypes } \\
\text { with wt/mut and mut/mut + mut/wt ( } \mathrm{p}= \\
0.008 \text { and } \mathrm{p}=0.02 \text {, respectively) }[124]\end{array}$ \\
\hline CYP2D6 & $\begin{array}{l}\text { CYP2D } 6 * 1, * 41, * 3, * 4, * 5 \\
* 1 \mathrm{xn},{ }^{*} 2 \mathrm{xn}\end{array}$ & $\begin{array}{l}\text { cross-sectional } \\
\geq 12 \text { months }\end{array}$ & $\begin{array}{l}\text { FGAs; equivalent } \\
\geq 100 \mathrm{mg} \\
\text { chlorpromazine } \\
\text { daily }\end{array}$ & SCZ; $n=66 ;$ European-Caucasian & $\begin{array}{l}\text { significant association between a greater } \\
\text { ability to metabolize CYP2D6, as measured by } \\
\text { increasing number of functional alleles, and } \\
\text { tardive dyskinesia }\left(\chi^{2}=7.65 \text {, d.f. }=1, p=\right. \\
0.006)[125]\end{array}$ \\
\hline CYP2D6 & $\begin{array}{l}\text { CYP } 2 \text { D } 6 * 1, * 3, * 4, * 10, * 41 \\
* 1 \mathrm{xn},{ }^{*} 2 \mathrm{xn}\end{array}$ & $\begin{array}{l}\text { clinical trial } \\
8 \text { days }\end{array}$ & risperidone (50) & $\begin{array}{l}\text { SCZ (43), SA (7); n = } 50(39 / 11) ; \\
\text { Caucasian }\end{array}$ & $\begin{array}{l}\text { indirect association suggesting that PMs and } \\
\text { IMs experienced a higher occurrence of EPS } \\
\text { in response to risperidone [128] }\end{array}$ \\
\hline CYP2D6 & CYP2D6* $1, * 4, * 5, * 6, * 1 x n$ & $\begin{array}{l}\text { RCT } \\
24 \text { h ( } 2 \times ; 2 \text { studies })\end{array}$ & $\begin{array}{l}\text { haloperidol (25) } \\
\text { risperidone (25) }\end{array}$ & $\begin{array}{l}\text { healthy volunteers, } \mathrm{n}=25(17 / 8) \\
\text { PMs, } \mathrm{n}=8 ; \mathrm{EMs}, \mathrm{n}=10 ; \mathrm{UMs}, \mathrm{n}=7 \text {; } \\
\text { Caucasian }\end{array}$ & $\begin{array}{l}\text { metabolizer status associated with differences } \\
\text { in 'EPS' (measured as wakefulness activity by } \\
\text { actigraphy) }[126,127]\end{array}$ \\
\hline CYP2D6 & unclear & cross-sectional & unclear & $\begin{array}{l}\text { SCZ (CATIE subsample); } \mathrm{n}=710 \\
(524 / 186)\end{array}$ & nonsignificant [129] \\
\hline CYP2D6 & ${ }^{*} 1, * 3, * 4, * 5, * 6, * 2 \mathrm{xN}$ & $\begin{array}{l}\text { retrospective } \\
8 \text { weeks }\end{array}$ & risperidone (83) & $\begin{array}{l}\text { first-episode SCZ, drug-naïve; } \mathrm{n}=83 \\
(17 / 66)\end{array}$ & nonsignificant [130] \\
\hline CYP2D6 & $\begin{array}{l}* 1, * 2,{ }^{*} 4, * 5, * 6 \mathrm{~B}, * 10 \mathrm{~B}, * 17 \\
* 29, * 35, * 41, * 43,{ }^{*} 106\end{array}$ & naturalistic cohort & risperidone (25) & $\begin{array}{l}\text { multiple diagnoses (unstated); } \mathrm{n}=24 \\
(16 / 8) ; \text { South-African Black (9), } \\
\text { White (15) }\end{array}$ & nonsignificant [131] \\
\hline$D P P 6$ & rs6977820 & $\begin{array}{l}\text { discovery: GWAS } \\
\text { replication: cohort }\end{array}$ & various & $\begin{array}{l}\mathrm{SCZ} \text {, discovery, } \mathrm{TD}, \mathrm{n}=61(35 / 26) ; \\
\text { non-TD, } \mathrm{n}=61(35 / 26) ; \text { replication, } \\
\mathrm{TD}, \mathrm{n}=36(18 / 18) ; \text { non-TD, } \mathrm{n}=136 \\
(88 / 50) ; \text { all Japanese }\end{array}$ & $\begin{array}{l}\text { discovery: } \mathrm{p}=7.0 \times 10^{-6}(<\text { reach genome- } \\
\text { wide significance); significant association in } \\
\text { replication sample: } \mathrm{p}=0.008 \text { (after correction } \\
\text { for multiple testing); combined: } \mathrm{p}=4.6 \times 10^{-6} \\
{[149]}\end{array}$ \\
\hline DRD2 & $\begin{array}{l}\text { rs6277, rs1800497 (Taq1A); } \\
\text { rs1800498 (Taq1D); } \\
\text { rs1799732 (-141CIns/Del) }\end{array}$ & $\begin{array}{l}\text { cross-sectional } \\
\geq 1 \text { month }\end{array}$ & $\begin{array}{l}\text { FGAs (37) } \\
\text { SGAs (303) } \\
\text { both (15) } \\
\text { missing }(47)\end{array}$ & $\begin{array}{l}\text { SCZ }(277) \text {, SA }(55) \text {, other PDs }(70) \\
\mathrm{n}=401 ; \text { relatively young (median } \\
\text { age }=26 \text { years) Caucasian sample }\end{array}$ & $\begin{array}{l}\text { association of Taq1A with TD nonsignificant, } \\
\text { though this variant was significantly } \\
\text { associated with akathisia }(\mathrm{p}=0.001) \text {; the } \\
-141 \mathrm{C} \text { variant was significantly associated } \\
\text { with TD }(\mathrm{p}=0.001)[139]\end{array}$ \\
\hline DRD2 & $\begin{array}{l}\text { rs6275, rs1800497 (Taq1A) } \\
\text { rs1800498 rs1801028 }\end{array}$ & $\begin{array}{l}\text { cross-sectional } \\
\text { at least } 3 \text { months }\end{array}$ & unclear & SCZ; n = $263(140 / 123) ;$ Korean & nonsignificant [139] \\
\hline HSPG2 & rs2445142 (G/A) & $\begin{array}{l}\text { discovery: GWAS } \\
\text { replication: cohort }\end{array}$ & various & $\begin{array}{l}\text { SCZ, discovery, } \mathrm{n}=50 \mathrm{TD}, \mathrm{n}=50 \\
\text { non-TD; replication, } \mathrm{n}=36 \mathrm{TD} \\
(18 / 18), \mathrm{n}=136 \text { non-TD }(88 / 50) \text {; all } \\
\text { Japanese }\end{array}$ & $\begin{array}{l}\text { nominally significant association with TD } \\
\text { attained in genome-wide and replication } \\
\text { samples }(\mathrm{p}=0.001 \text { and } \mathrm{p}=0.002 \\
\text { respectively); combined } \mathrm{p}=2.0 \times 10^{-5}[145]\end{array}$ \\
\hline HSPG2 & $\begin{array}{l}\text { rs2445142 (G/A) rs878949 } \\
\left(\text { surrogate; } r^{2}=1\right)\end{array}$ & $\begin{array}{l}\text { CATIE: prospective } \\
\text { Jewish: cross-sectional } \\
\geq 3 \text { months }\end{array}$ & various & $\begin{array}{l}\text { Jewish-Israeli sample, } \mathrm{n}=166 \\
(89 / 77) \text {; CATIE subsample, } \mathrm{n}=179 \\
(147 / 32) ; \text { European-Caucasian }\end{array}$ & $\begin{array}{l}\text { nominal significant association identified } \\
\text { between rs } 2445142 \text { (or rs } 878949 \text { as a } \\
\text { surrogate) in both samples ( } \mathrm{p}=0.003 \text { and } \mathrm{p}= \\
0.039 \text {, respectively), with the } \mathrm{G} \text { allele being the } \\
\text { risk allele [146] }\end{array}$ \\
\hline HSPG2 & rs2445142, rs2270697 & prospective & unclear & $\begin{array}{l}\text { most SCZ, PD, or other; } \mathrm{n}=168 \text {; } \\
\text { Caucasian }\end{array}$ & nonsignificant, including rs2445142 [147] \\
\hline SLC18A2 & rs2015586 & cross-sectional & unclear & $\begin{array}{l}\text { SCZ (CATIE subsample); } \mathrm{n}=710 \\
(524 / 186) ; 56 \% \text { European-American }\end{array}$ & $\begin{array}{l}\mathrm{p}=9.858 \times 10^{-5} \text {; result is nonsignificant at } \\
\text { genome-wide significance of } 5.0 \times 10^{-8}[129]\end{array}$ \\
\hline $\begin{array}{l}S L C 18 A 2 \\
\text { and } \\
D R D 2\end{array}$ & $\begin{array}{l}\text { SLC18A2: rs2015586, } \\
\text { rs363224 DRD2: rs6277 }\end{array}$ & $\begin{array}{l}\text { retrospective } \\
\text { variable durations }\end{array}$ & various & $\begin{array}{l}\text { SCZ or SCZ spectrum disorder; } \mathrm{n}= \\
\text { 223; Caucasian (193), African- } \\
\text { American (30) }\end{array}$ & $\begin{array}{l}\text { gene-gene interaction involving C allele of } \\
\text { rs363224 and the C allele of rs6277 was } \\
\text { significantly associated with AIMS scores } \\
(p=0.001) \text {; nominal association involving } \\
\text { rs2015586 [140] }\end{array}$ \\
\hline
\end{tabular}

AIMS = Abnormal Involuntary Movements Scale; mut = mutant; PD = psychotic disorder; RCT = randomized controlled trial; SA = schizoaffective disorder; UM = ultrarapid metabolizer; wt = wild type.

Genetics of Common AntipsychoticInduced Adverse Effects
Mol Neuropsychiatry 2016;2:61-78

DOI: 10.1159/000445802 
A large-scale candidate gene study utilizing data from the CATIE sample, however, found no association between TD and CYP2D6 [129]. Interestingly, results from a recent cross-sectional study by Koola et al. [125] suggested that the risk of TD was positively correlated with the number of functional CYP2D6 alleles that an individual carries. The experimenters speculated that active metabolites of FGAs might have toxic pharmacodynamic properties, and that a greater capacity to metabolize FGAs could therefore enhance susceptibility to TD by increasing exposure to these toxic metabolites. Three other studies involving healthy volunteers reported an indirect association between the PM or IM CYP2D6 phenotypes and a greater risk of developing EPS [126-128]. Finally, 2 studies involving risperidone-treated samples found no association between CYP2D6 metabolizer status and EPS $[130,131]$.

Owing to difficulties in characterizing CYP2D6 genotypes, most studies investigating associations with this gene have limited sample sizes and are therefore underpowered. Also, naturalistic and cross-sectional pharmacogenetic studies of CYP2D6 are limited given that numerous factors influencing the activity of this enzyme (e.g., co-medications, diet) remain unaccounted for. The meta-analysis performed by Fleeman et al. [124], which yielded significant results only after excluding cross-sectional and retrospective studies, underscores this point. Accordingly, a greater number of prospective studies, with sample sizes providing adequate power, are needed to clarify the role of CYP2D6 in AP-induced EPS/TD.

\section{Dopamine Receptor $\mathrm{D}_{2}$}

The binding of dopamine $\mathrm{D}_{2}$ receptors (DRD2) is hallmark feature of all APs and is strongly linked to their efficacy in treating the positive symptoms associated with SCZ and related spectrum disorders [132]. Because dopaminergic transmission in the nigrostriatal pathway is essential for adaptive motor control [133], aberrant DA signaling in this pathway is thought to underlie - at least in part - the pathophysiology of TD and EPS [134, 135]. In light of this, various studies have investigated the possibility that variation at the DRD2 locus could explain individual differences in susceptibility to AP-induced EPS and TD. The DRD2/ANKK1 marker rs1800497 (TaqIA) has yielded the most consistent findings, with two metaanalyses conducted prior to 2010 supporting an association with TD $[136,137]$. However, the two studies included in our review that reexamined this association yielded negative results $[138,139]$. Nevertheless, these studies hold little weight in view of the overall evidence, and the association between the TaqIA variant and TD susceptibility continues to be of clinical interest. Future studies should aim to investigate the clinical utility of this variant in guiding treatment selection.

\section{SLC18A2}

The SLC18A2 gene encodes vesicular monoamine transporter 2 (VMAT2), which is involved in regulating the release of numerous neurotransmitters, including dopamine. A recent study by our lab revealed a significant association between the SLC18A2 rs363224 CC genotype and susceptibility to TD in sample of chronic SCZ patients [140]. An association of TD with an interaction between the SLC18A2 rs363224 C allele and the putatively functional $D R D 2$ rs6277 C allele showed an even greater effect size. Several nominally significant results for SLC18A2 were also reported, the most interesting of which was rs2015586. This SNP was the top signal detected by Tsai et al. [129] in their candidate gene study of TD in the CATIE subsample.

\section{HSPG2}

HSPG2 encodes heparan sulfate proteoglycan 2, or perlecan, a highly conserved and essential structural protein originally identified within the basal lamina (i.e. basement membrane) [141]. Perlecan has also been shown to have an important role in endocytosis, as well as in the mediation of cell signaling, migration and proliferation [142, 143].

Using a genome-wide approach, a team of Japanese researchers [144] identified a number of putative associations predisposing to treatment-resistant TD, with the strongest signal emerging from an intronic SNP (rs2445142) located in the HSPG2 gene. Although none of the associations survived correction for multiple testing, a replication study reanalyzing the top 67 hits in an independent sample was performed [145]. Case and control criteria were stringently defined so as to represent an extreme distribution of the TD phenotype, thereby increasing the power of the sample. A nominally significant association between the HSPG2 SNP rs2445142 and TD was once again detected. As with the discovery sample, the $G$ allele was found to be overrepresented in the treatment-resistant group. Pooling the results from genomewide and replication samples, the rs2445142-TD association attained an allelic p value of $2.0 \times 10^{-5}$. Functional studies of the rs 2445142 variant provided further evidence that HSPG2 is involved in TD [145]. Moreover, this association was later replicated by Greenbaum et al. [146] in two independent samples, one of Jewish-Israeli ances-
72

Mol Neuropsychiatry 2016;2:61-78 DOI: $10.1159 / 000445802$
MacNeil/Müller 
try and the other of American-European ancestry. After limiting the control group of the Jewish-Israeli sample to subjects representing an 'extreme' TD-resistant phenotype, a nominally significant association between the risk rs2445142 G allele and TD was detected. A nominal association was also identified in the European-American sample using a surrogate marker $\left(\mathrm{rs} 878949, \mathrm{r}^{2}=1\right)$ as a proxy for the rs2445142 genotype. A recent prospective study by Bakker et al. [147], however, was unable to verify this association. Still, given the naturalistic design of this study, it is possible that the signal was masked. While the results for HSPG2 are promising, further replication of this finding is warranted.

\section{DPP6}

DPP6 encodes dipeptidyl peptidase protein 6, an auxiliary subunit of kv4.2 voltage-gated potassium channels [148]. Using the same discovery sample and a similar methodological approach to that of the Japanese study on HSPG2 (though this time utilizing a different SNP array), the same research team detected an association between the DPP6 intronic SNP rs6977820 and TD [149]. The A allele was found to be overrepresented in treatment-resistant TD cases. However, the association did not reach a genome-wide level of significance (defined at $\mathrm{p}<1.9 \times$ $10^{7}$ ). Nevertheless, replication in an independent sample yielded a significant result (allelic $\mathrm{p}=0.008$ ), giving a combined sample $\mathrm{p}$ value of $4.6 \times 10^{6}$. Subsequent functional studies conducted by the investigators provided further evidence that the DPP6 SNP rs6977820 influences susceptibility to TD [149]. In sum, DPP6 appears to be a promising biomarker for identifying patients at risk of developing treatment-resistant TD.

\section{Discussion}

In this review, we surveyed the literature on the PGx of AAEs from 2010 to 2015 inclusive, placing emphasis on independently replicated gene-drug associations that have been supported by expert panels. The studies included in the review assessed genetic associations involving metabolic dysregulation and movement disorders.

With respect to metabolic dysregulation, the studies included in this review have reaffirmed associations of the HTR2C $-759 \mathrm{C} / \mathrm{T}$ and LEP -2548G/A SNPs with AIWG [e.g., 45, 74]. Furthermore, additional evidence has accumulated in support of an association between the HTR2C rs1414334 SNP and AP-MetS [49]. With respect to AIWG, the MC4R marker rs 489693 arguably represents the most promising finding to have emerged within the last 5 years $[75,93,96,97]$. Also, a recent GWAS has identified a promising biomarker of AIWG risk at the OGFRL1 locus [115].

In terms of TD and EPS, a large meta-analysis has provided additional support for an association between abnormal CYP2D6 metabolizer status and greater susceptibility to AP-induced movement disorders, namely AIP and TD [124]. Research has also implicated variants of the SLC18A2 gene and an interaction between SLC18A2 and $D R D 2$ in TD susceptibility $[129,140]$. Finally, HSPG2 and $D P P 6$ have emerged as promising candidates for the prediction of TD susceptibility $[145,146,149]$.

The studies on HSPG2 and DPP6 are noteworthy for reasons other than the results they obtained. The studies that looked at these genes involved a stringent characterization of the phenotypes constituting cases and controls. Enhancements in power attained through extreme phenotype sampling can help to facilitate the identification of risk alleles that are relatively rare or that only exert a modest effect on drug-gene phenotypes [150]. More frequent application of this design strategy could benefit psychiatric PGx by identifying variants that might usually go undetected due to noise within the sample. However, the limitations inherent to this sampling method, such as the requirement for more extensive screening protocols and the potential that detected variant effects may not apply to a broader distribution of the phenotype [151], need to be considered.

Also of interest in this review was the number of studies representing cases in which the consideration of multilocus effects yielded significant findings, while the examination of single markers was shown to yield nominal or nonsignificant results, or significant results with otherwise smaller effect sizes [46, 72, 75, 107, 140]. These studies highlight the genetic complexity of the phenotypes under examination and the need for investigators to place greater emphasis on gene-gene interactions in unravelling the pharmacogenetic determinants of AAEs. Statistical tools and methods for performing analyses of this complexity are becoming increasingly more efficient [152-154]. For example, principles of pathway-analysis are being combined with machine learning techniques in order to detect epistatic effects from high-dimensional GWAS datasets [155]. The application of computerized algorithms including genetic and nongenetic variables to predict AAEs appears to be particularly promising [e.g., 156]. 


\section{Conclusion}

In summary, pharmacogenetic studies of AAEs bear the promise of improving treatment outcomes by allowing physicians to deliver personalized treatments with minimal AEs and optimal efficacy. One important aspect is that gene variants frequently have larger effect sizes in pharmacogenetic phenotypes compared with complex disease risk [157]. Consistent with this, gene variants associated with AIWG appear to have relatively large effect sizes, especially the rs489693 marker at the MC4R gene locus [93]. Furthermore, there is general consensus for an association between CYP2D6 abnormal metabolizer status and an increased risk of AAEs, including TD and parkinsonism [124]. The levels of evidence assigned to AAE genetic associations by expert clinical PGx panels and consortia have continued to increase. In addition, drug regulatory agencies, such as the FDA and Health Canada, are beginning to include PGx information on AP drug labels. Nevertheless, the current level of evidence remains limited, and further validation by additional studies is required. Also, further research is needed to identify novel and interacting variants for AAEs. Building up on these efforts will undoubtedly lead to the implementation of genetic testing to predict and thereby reduce the occurrence of AAEs.

\section{Disclosure Statement}

The authors declare no conflicts of interest.

\section{References}

1 Muralidharan K, Ali M, Silveira LE, Bond DJ, Fountoulakis KN, Lam RW, et al: Efficacy of second generation antipsychotics in treating acute mixed episodes in bipolar disorder: a meta-analysis of placebo-controlled trials. J Affect Disord 2013;150:408-414

2 Zhou XP, Keitner GIP, Qin BM, Ravindran AVP, Bauer MP, Del Giovane CP, et al: Atypical antipsychotic augmentation for treatment-resistant depression: a systematic review and network meta-analysis. Int J Neuropsychopharmacol DOI: 10.1093/ijnp/pyv060.

3 McCracken JT, McGough J, Shah B, Cronin $\mathrm{P}$, Hong $\mathrm{D}$, Aman MG, et al: Risperidone in children with autism and serious behavioral problems. N Engl J Med 2002;347:314-321.

4 Zádori D, Veres G, Szalárdy L, Klivényi P, Vécsei L: Drug-induced movement disorders. Expert Opin Drug Saf 2015;14:877-890.

5 Jeste DV, Caligiuri MP: Tardive dyskinesia. Schizophr Bull 1993;19:303-315.

6 Hartling L, Abou-Setta AM, Dursun S, Mousavi SS, Pasichnyk D, Newton AS: Antipsychotics in adults with schizophrenia: comparative effectiveness of first-generation versus second-generation medications: a systematic review and meta-analysis. Ann Intern Med 2012;157:498-511.

7 Leucht S, Cipriani A, Spineli L, Mavridis D, Orey D, Richter F, et al: Comparative efficacy and tolerability of 15 antipsychotic drugs in schizophrenia: a multiple-treatments metaanalysis. Lancet 2013;382:951-962.

8 De Hert M, Detraux J, van Winkel R, Yu W, Correll CU: Metabolic and cardiovascular adverse effects associated with antipsychotic drugs. Nat Rev Endocrinol 2012;8:114-126.

9 Strom BL, Eng SM, Faich G, Reynolds RF, D’Agostino RB, Ruskin J, et al: Comparative mortality associated with ziprasidone and olanzapine in real-world use among 18,154 patients with schizophrenia: the Ziprasidone Observational Study of Cardiac Outcomes (ZODIAC). Am J Psychiatry 2011;168:193201.

10 Nagaya T, Yoshida H, Takahashi H, Kawai M: Heart rate-corrected QT interval in resting ECG predicts the risk for development of type-2 diabetes mellitus. Eur J Epidemiol 2010;25:195-202.

11 Lieberman JA, Stroup TS, McEvoy JP, Swartz MS, Rosenheck RA, Perkins DO, et al: Effectiveness of antipsychotic drugs in patients with chronic schizophrenia. N Engl J Med 2005;353:1209-1223.

12 Ascher-Svanum H, Faries DE, Zhu B, Ernst FR, Swartz MS, Swanson JW: Medication adherence and long-term functional outcomes in the treatment of schizophrenia in usual care. J Clin Psychiatry 2006;67:453-460.

13 Tafesse E, Hines PL, Carson WH: Atypical antipsychotic adherence and hospitalization in patients with schizophrenia. Schizophr Res 2003;60:346-346.

14 Novick D, Haro JM, Suarez D, Perez V, Dittmann RW, Haddad PM: Predictors and clinical consequences of non-adherence with antipsychotic medication in the outpatient treatment of schizophrenia. Psychiatry Res 2010;176:109-113.

15 Müller DJ, Schulze TG, Knapp M, Held T, Krauss H, Weber T, et al: Familial occurrence of tardive dyskinesia. Acta Psychiatr Scand 2001;104:375-379.

16 Gebhardt S, Theisen FM, Haberhausen M, Heinzel-Gutenbrunner M, Wehmeier PM, Krieg JC, et al: Body weight gain induced by atypical antipsychotics: an extension of the monozygotic twin and sib pair study. J Clin Pharm Ther 2010;35:207-211.

17 Changasi AH, Shams TA, Pouget JG, Müller DJ: Genetics of antipsychotic drug outcome and implications for the clinician: into the limelight. Transl Dev Psychiatry 2014;2:24663.

18 Caudle KE, Klein TE, Hoffman JM, Müller DJ, Whirl-Carrillo M, Gong L, et al: Incorporation of pharmacogenomics into routine clinical practice: the Clinical Pharmacogenetics Implementation Consortium (CPIC) guideline development process. Curr Drug Metab 2014;15:209-217.

19 Whirl-Carrillo M, McDonagh EM, Hebert JM, Gong L, Sangkuhl K, Thorn CF, et al: Pharmacogenomics knowledge for personalized medicine. Clin Pharmacol Ther 2012;92: 414-417.

20 Relling MV, Klein TE: CPIC: Clinical Pharmacogenetics Implementation Consortium of the Pharmacogenomics Research Network. Clin Pharmacol Ther 2011;89:464-467.

21 Hicks JK, Bishop JR, Sangkuhl K, Muller DJ, Ji Y, Leckband SG, et al: Clinical Pharmacogenetics Implementation Consortium (CPIC) guideline for CYP2D6 and CYP2C19 genotypes and dosing of selective serotonin reuptake inhibitors. Clin Pharmacol Ther 2015;98: 127-134.

22 Swen JJ, Nijenhuis M, de Boer A, Grandia L, Maitland-van der Zee AH, Mulder $\mathrm{H}$, et al: Pharmacogenetics: from bench to byte - an update of guidelines. Clin Pharmacol Ther 2011;89:662-673.

23 Fagerness J, Fonseca E, Hess G, Scott R, Gardner $\mathrm{K}$, Koffler $\mathrm{M}$, et al: Pharmacogeneticguided psychiatric intervention associated with increased adherence and cost savings. Am J Manag Care 2014:e146-e156. 
24 Müller DJ, Kekin I, Kao AC, Brandl EJ: Towards the implementation of CYP2D6 and CYP2C19 genotypes in clinical practice: update and report from a pharmacogenetic service clinic. Int Rev Psychiatry 2013;25:554-571.

25 Walden LM, Brandl EJ, Changasi A, Sturgess JE, Soibel A, Notario JF, et al: Physicians' opinions following pharmacogenetic testing for psychotropic medication. Psychiatry Res 2015;229:913-918.

26 Benitez J, Jablonski MR, Allen JD, Winner JG: The clinical validity and utility of combinatorial pharmacogenomics: enhancing patient outcomes. Appl Transl Genomics 2015;5:4749.

27 Altar CA, Carhart J, Josiah AD, Hall-Flavin D, Winner JG, Dechairo B: Clinical utility of combinatorial pharmacogenomics-guided antidepressant therapy: evidence from three clinical studies. Mol Neuropsychiatry 2015;1: 145-155.

28 Lett TA, Wallace TJ, Chowdhury NI, Tiwari AK, Kennedy JL, Müller DJ: Pharmacogenetics of antipsychotic-induced weight gain: review and clinical implications. Mol Psychiatry 2012;17:242-266.

29 Ravyn D, Ravyn V, Lowney R, Nasrallah HA: CYP450 pharmacogenetic treatment strategies for antipsychotics: a review of the evidence. Schizophr Res 2013;149:1-14.

30 Arranz MJ, Rivera M, Munro JC: Pharmacogenetics of response to antipsychotics in patients with schizophrenia. CNS Drugs 2011; 25:933-969.

31 Lencz T, Malhotra AK: Pharmacogenetics of antipsychotic-induced side effects. Dialogues Clin Neurosci 2009;11:405-415.

32 Müller DJ, Kennedy JL: Genetics of antipsychotic treatment emergent weight gain in schizophrenia. Pharmacogenomics 2006;7: 863-887.

$33 \mathrm{Xu}$ Y, Jones JE, Kohno D, Williams KW, Lee CE, Choi MJ, et al: 5-HT2CRs expressed by pro-opiomelanocortin neurons regulate energy homeostasis. Neuron 2008;60:582-589.

34 Zhou L, Sutton GM, Rochford JJ, Semple RK, Lam DD, Oksanen Laura J, et al: Serotonin 2C receptor agonists improve type 2 diabetes via melanocortin-4 receptor signaling pathways. Cell Metab 2007;6:398-405.

35 Donovan MH, Tecott LH: Serotonin and the regulation of mammalian energy balance. Front Neurosci 2013;7:36.

36 Meltzer HY: The role of serotonin in antipsychotic drug action. Neuropsychopharmacology 1999;21:106S-115S.

37 De Luca V, Müller DJ, de Bartolomeis A, Kennedy JL: Association of the HTR2C gene and antipsychotic induced weight gain: a metaanalysis. Int J Neuropsychopharmacol 2007; 10:697-704.

38 Buckland PR, Hoogendoorn B, Guy CA, Smith SK, Coleman SL, O'Donovan MC: Low gene expression conferred by association of an allele of the 5-HT2C receptor gene with antipsychotic-induced weight gain. Am J Psychiatry 2005;162:613-615.
39 Hill MJ, Reynolds GP: 5-HT2C receptor gene polymorphisms associated with antipsychotic drug action alter promoter activity. Brain Res 2007;1149:14-17.

40 Hill MJ, Reynolds GP: Functional consequences of two HTR2C polymorphisms associated with antipsychotic-induced weight gain. Pharmacogenomics 2011;12:727-734.

41 Wallace TJ, Zai CC, Brandl EJ, Müller DJ: Role of 5-HT(2C) receptor gene variants in antipsychotic-induced weight gain. Pharmacogenomics Pers Med 2011;4:83-93.

42 Laika B, Leucht S, Heres S, Schneider H, Steimer W: Pharmacogenetics and olanzapine treatment: CYP1A2* $1 \mathrm{~F}$ and serotonergic polymorphisms influence therapeutic outcome. Pharmacogenomics J 2010;10:20-29.

43 Hoekstra PJ, Troost PW, Lahuis BE, Mulder $\mathrm{H}$, Mulder EJ, Franke B, et al: Risperidoneinduced weight gain in referred children with autism spectrum disorders is associated with a common polymorphism in the 5-hydroxytryptamine $2 \mathrm{C}$ receptor gene. J Child Adolesc Psychopharmacol 2010;20:473-477.

44 Opgen-Rhein C, Brandl EJ, Müller DJ, Neuhaus AH, Tiwari AK, Sander T, et al: Association of HTR2C, but not LEP or INSIG2, genes with antipsychotic-induced weight gain in a German sample. Pharmacogenomics 2010; 11:773-780.

45 Sicard MN, Zai CC, Tiwari AK, Souza RP, Meltzer HY, Lieberman JA, et al: Polymorphisms of the HTR2C gene and antipsychotic-induced weight gain: an update and metaanalysis. Pharmacogenomics 2010;11:15611571.

46 Gregoor JG, Mulder H, Cohen D, van Megen HJGM, Egberts TCG, Heerdink ER, et al: Combined HTR2C-LEP genotype as a determinant of obesity in patients using antipsychotic medication. J Clin Psychopharmacol 2010;30:702-705.

47 Correia CT, Almeida JP, Santos PE, Sequeira AF, Marques CE, Miguel TS, et al: Pharmacogenetics of risperidone therapy in autism: association analysis of eight candidate genes with drug efficacy and adverse drug reactions. Pharmacogenomics J 2010;10:418-430.

48 Del Castillo N, Zimmerman MB, Tyler B, Ellingrod VL, Calarge C: $759 \mathrm{C} / \mathrm{T}$ variants of the serotonin (5-HT2C) receptor gene and weight gain in children and adolescents in long-term risperidone treatment. Clin Pharmacol Biopharm 2013;2:110.

49 Ma X, Maimaitirexiati T, Zhang R, Gui X, Zhang W, Xu G, et al: HTR2C polymorphisms, olanzapine-induced weight gain and antipsychotic-induced metabolic syndrome in schizophrenia patients: a metaanalysis. Int J Psychiatry Clin Pract 2014;18: 229-242.

50 Kang SH, Lee JI, Han HR, Soh M, Hong JP: Polymorphisms of the leptin and HTR2C genes and clozapine-induced weight change and baseline BMI in patients with chronic schizophrenia. Psychiatr Genet 2014;24:249256.
51 Thompson A, Lavedan C, Volpi S: Absence of weight gain association with the HTR2C $-759 \mathrm{C} / \mathrm{T}$ polymorphism in patients with schizophrenia treated with iloperidone. Psychiatry Res 2010;175:271-273.

52 Gregoor JG, van der Weide J, Loovers HM, van Megen HJ, Egberts TC, Heerdink ER: Polymorphisms of the LEP, LEPR and HTR2C gene: obesity and BMI change in patients using antipsychotic medication in a naturalistic setting. Pharmacogenomics 2011;12:919923

53 Houston JP, Kohler J, Bishop JR, Ellingrod VL, Ostbye KM, Zhao F, et al: Pharmacogenomic associations with weight gain in olanzapine treatment of patients without schizophrenia. J Clin Psychiatry 2012;73:10771086.

54 Kuzman MR, Medved V, Bozina N, Grubisin J, Jovanovic N, Sertic J: Association study of MDR1 and 5-HT2C genetic polymorphisms and antipsychotic-induced metabolic disturbances in female patients with schizophrenia. Pharmacogenomics J 2011;11:35-44.

55 Almoguera B, Riveiro-Alvarez R, Lopez-Castroman J, Dorado P, Vaquero-Lorenzo C, Fernandez-Piqueras J, et al: Association of common genetic variants with risperidone adverse events in a Spanish schizophrenic population. Pharmacogenomics J 2013;13: 197-204.

56 Burns CM, Chu H, Rueter SM, Hutchinson LK, Canton H, Sanders-Bush E, et al: Regulation of serotonin-2C receptor G-protein coupling by RNA editing. Nature 1997;387:303308

57 Drago A, Serretti A: Focus on HTR2C: a possible suggestion for genetic studies of complex disorders. Am J Med Genet B Neuropsychiatr Genet 2009;150B:601-637.

58 Lin PI, Vance JM, Pericak-Vance MA, Martin ER: No gene is an island: the flip-flop phenomenon. Am J Hum Genet 2007;80:531538

59 Mulder H, Cohen D, Scheffer H, Gispen-de Wied C, Arends J, Wilmink FW, et al: HTR2C gene polymorphisms and the metabolic syndrome in patients with schizophrenia: a replication study. J Clin Psychopharmacol 2009. 29:16-20.

60 Mulder H, Franke B, van der-Beek van der AA, Arends J, Wilmink FW, Scheffer $\mathrm{H}$, et al: The association between HTR2C gene polymorphisms and the metabolic syndrome in patients with schizophrenia. J Clin Psychopharmacol 2007;27:338-343.

61 Risselada AJ, Vehof J, Bruggeman R, Wilffert B, Cohen D, Al Hadithy AF, Arends J, Mulder $\mathrm{H}$ : Association between HTR2C gene polymorphisms and the metabolic syndrome in patients using antipsychotics: a replication study. Pharmacogenomics J 2010;1:62-67. 
62 Klemettila JP, Kampman O, Seppala N, Viikki M, Hamalainen M, Moilanen E, et al: Association study of the HTR2C, leptin and adiponectin genes and serum marker analyses in clozapine treated long-term patients with schizophrenia. Eur Psychiatry 2015;30:296302.

63 Bai YM, Chen TT, Liou YJ, Hong CJ, Tsai SJ: Association between HTR2C polymorphisms and metabolic syndrome in patients with schizophrenia treated with atypical antipsychotics. Schizophr Res 2011;125:179-186.

64 Halaas JL, Gajiwala KS, Maffei M, Cohen SL, Chait BT, Rabinowitz D, et al: Weight-reducing effects of the plasma protein encoded by the obese gene. Science 1995;269:543-546.

65 Myers MG Jr, Olson DP: Central nervous system control of metabolism. Nature 2012;491: 357-363.

66 Bingham NC, Anderson KK, Reuter AL, Stallings NR, Parker KL: Selective loss of leptin receptors in the ventromedial hypothalamic nucleus results in increased adiposity and a metabolic syndrome. Endocrinology 2008; 149:2138-2148.

67 Clement K, Vaisse C, Lahlou N, Cabrol S, Pelloux V, Cassuto D, et al: A mutation in the human leptin receptor gene causes obesity and pituitary dysfunction. Nature 1998;392: 398-401.

68 Wabitsch M, Funcke J-B, Lennerz B, KuhnleKrahl U, Lahr G, Debatin K-M, et al: Biologically inactive leptin and early-onset extreme obesity. New Engl J Med 2015;372:48-54.

69 Mammes O, Betoulle D, Aubert R, Herbeth B, Siest G, Fumeron F: Association of the G2548A polymorphism in the $5^{\prime}$ region of the LEP gene with overweight. Ann Hum Genet 2000;64:391-394.

70 Li WD, Reed DR, Lee JH, Xu W, Kilker RL, Sodam BR, et al: Sequence variants in the $5^{\prime}$ flanking region of the leptin gene are associated with obesity in women. Ann Hum Genet 1999;63:227-234.

71 Yu Z, Han S, Cao X, Zhu C, Wang X, Guo X: Genetic polymorphisms in adipokine genes and the risk of obesity: a systematic review and meta-analysis. Obesity 2011;20:396-406.

72 Yevtushenko OO, Cooper SJ, O’Neill R, Doherty JK, Woodside JV, Reynolds GP: Influence of 5-HT2C receptor and leptin gene polymorphisms, smoking and drug treatment on metabolic disturbances in patients with schizophrenia. Br J Psychiatry 2008;192:424428.

73 Kuo PH, Kao CF, Chen PY, Chen CH, Tsai YS, Lu ML, et al: Polymorphisms of INSIG2, MC4R, and LEP are associated with obesityand metabolic-related traits in schizophrenic patients. J Clin Psychopharmacol 2011;31: 705-711.

74 Brandl EJ, Frydrychowicz C, Tiwari AK, Lett TA, Kitzrow W, Buttner S, et al: Association study of polymorphisms in leptin and leptin receptor genes with antipsychotic-induced body weight gain. Prog Neuropsychopharmacol Biol Psychiatry 2012;38:134-141.
75 Nurmi EL, Spilman SL, Whelan F, Scahill LL, Aman MG, McDougle CJ, et al: Moderation of antipsychotic-induced weight gain by energy balance gene variants in the RUPP autism network risperidone studies. Transl Psychiatry 2013;3:e274.

76 Fernandez E, Carrizo E, Fernandez V, Connell L, Sandia I, Prieto D, et al: Polymorphisms of the LEP- and LEPR genes, metabolic profile after prolonged clozapine administration and response to the antidiabetic metformin. Schizophr Res 2010;121:213217.

77 Perez-Iglesias R, Mata I, Amado JA, Berja A, Garcia-Unzueta MT, Martinez Garcia O, et al: Effect of FTO, SH2B1, LEP, and LEPR polymorphisms on weight gain associated with antipsychotic treatment. J Clin Psychopharmacol 2010;30:661-666

78 Gregoor JG, van der Weide J, Mulder H, Cohen D, van Megen HJ, Egberts AC, et al: Polymorphisms of the LEP- and LEPR gene and obesity in patients using antipsychotic medication. J Clin Psychopharmacol 2009;29:2125.

79 Tiwari AK, Zai CC, Likhodi O, Lisker A, Singh D, Souza RP, et al: A common polymorphism in the cannabinoid receptor 1 (CNR1) gene is associated with antipsychotic-induced weight gain in schizophrenia. Neuropsychopharmacology 2010;35:1315-1324.

80 Gregoor JG, van der Weide J, Loovers HM, van Megen HJ, Egberts TC, Heerdink ER: Association between LEP and LEPR gene polymorphisms and dyslipidemia in patients using atypical antipsychotic medication. Psychiatr Genet 2010;20:311-316.

81 Roffeei SN, Mohamed Z, Reynolds GP, Said MA, Hatim A, Mohamed EH, et al: Association of FTO, LEPR and MTHFR gene polymorphisms with metabolic syndrome in schizophrenia patients receiving antipsychotics. Pharmacogenomics 2014;15:477-485.

82 Cone RD: Anatomy and regulation of the central melanocortin system. Nat Neurosci 2005; 8:571-578.

83 Van der Ploeg LH, Martin WJ, Howard AD, Nargund RP, Austin CP, Guan X, et al: A role for the melanocortin 4 receptor in sexual function. Proc Natl Acad Sci USA 2002;99: 11381-11386

84 Caruso C, Durand D, Schioth HB, Rey R, Seilicovich A, Lasaga M: Activation of melanocortin 4 receptors reduces the inflammatory response and prevents apoptosis induced by lipopolysaccharide and interferon-gamma in astrocytes. Endocrinology 2007;148:49184926.

85 Chu HC, Xia JL, Xu HM, Yang Z, Gao J, Liu $\mathrm{SH}$ : Melanocortin 4 receptor mediates neuropathic pain through p38MAPK in spinal cord. Can J Neurol Sci 2012;39:458-464.

86 Sohn JW, Harris LE, Berglund ED, Liu T, Vong L, Lowell BB, et al: Melanocortin 4 receptors reciprocally regulate sympathetic and parasympathetic preganglionic neurons. Cell 2013;152:612-619.
87 Mountjoy KG, Mortrud MT, Low MJ, Simerly RB, Cone RD: Localization of the melanocortin-4 receptor (MC4-R) in neuroendocrine and autonomic control circuits in the brain. Mol Endocrinol 1994;8:1298-1308.

88 Lam DD, Przydzial MJ, Ridley SH, Yeo GS, Rochford JJ, O'Rahilly S, et al: Serotonin 5-HT2C receptor agonist promotes hypophagia via downstream activation of melanocortin 4 receptors. Endocrinology 2008; 149: 1323-1328.

89 Seeley RJ, Yagaloff KA, Fisher SL, Burn P, Thiele TE, van Dijk G, et al: Melanocortin receptors in leptin effects. Nature 1997;390:349.

90 Huszar D, Lynch CA, Fairchild-Huntress V, Dunmore JH, Fang Q, Berkemeier LR, et al: Targeted disruption of the melanocortin-4 receptor results in obesity in mice. Cell 1997;88: 131-141.

91 Farooqi IS, Keogh JM, Yeo GS, Lank EJ, Cheetham T, O’Rahilly S: Clinical spectrum of obesity and mutations in the melanocortin 4 receptor gene. N Engl J Med 2003;348:10851095.

92 Yilmaz Z, Davis C, Loxton NJ, Kaplan AS, Levitan RD, Carter JC, et al: Association between MC4R rs17782313 polymorphism and overeating behaviors. Int J Obesity (Lond) 2015;39:114-120.

93 Malhotra AK, Correll CU, Chowdhury NI, Müller DJ, Gregersen PK, Lee AT, et al: Association between common variants near the melanocortin 4 receptor gene and severe antipsychotic drug-induced weight gain. Arch Gen Psychiatry 2012;69:904-912.

94 Loos RJ, Lindgren CM, Li S, Wheeler E, Zhao $\mathrm{JH}$, Prokopenko I, et al: Common variants near MC4R are associated with fat mass, weight and risk of obesity. Nat Genet 2008;40: 768-775.

95 Chambers JC, Elliott P, Zabaneh D, Zhang W, $\mathrm{Li} Y$, Froguel $\mathrm{P}$, et al: Common genetic variation near MC4R is associated with waist circumference and insulin resistance. Nat Genet 2008;40:716-718

96 Czerwensky F, Leucht S, Steimer W: MC4R rs489693: a clinical risk factor for second generation antipsychotic-related weight gain? Int J Neuropsychopharmacol 2013;16:2103-2109.

97 Chowdhury NI, Tiwari AK, Souza RP, Zai CC, Shaikh SA, Chen S, et al: Genetic association study between antipsychotic-induced weight gain and the melanocortin-4 receptor gene. Pharmacogenomics J 2013;13: 272-279.

98 Czerwensky F, Leucht S, Steimer W: Association of the common MC4R rs17782313 polymorphism with antipsychotic-related weight gain. J Clin Psychopharmacol 2013; 33:74-79.

99 Goyette P, Sumner JS, Milos R, Duncan AM, Rosenblatt DS, Matthews RG, et al: Human methylenetetrahydrofolate reductase: isolation of cDNA, mapping and mutation identification. Nat Genet 1994;7:195-200. 
100 Ueland PM, Hustad S, Schneede J, Refsum $\mathrm{H}$, Vollset SE: Biological and clinical implications of the MTHFR C677T polymorphism. Trends Pharmacol Sci 2001;22:195201.

101 Frosst P, Blom HJ, Milos R, Goyette P, Sheppard CA, Matthews RG, et al: A candidate genetic risk factor for vascular disease: a common mutation in methylenetetrahydrofolate reductase. Nat Genet 1995;10:111113.

102 van der Put NM, Gabreels F, Stevens EM, Smeitink JA, Trijbels FJ, Eskes TK, et al: A second common mutation in the methylenetetrahydrofolate reductase gene: an additional risk factor for neural-tube defects? Am J Hum Genet 1998;62:1044-1051.

103 Klerk M, Verhoef P, Clarke R, Blom HJ, Kok FJ, Schouten EG, et al: MTHFR 677C->T polymorphism and risk of coronary heart disease: a meta-analysis. JAMA 2002;288: 2023-2031.

104 Muntjewerff JW, Kahn RS, Blom HJ, den Heijer M: Homocysteine, methylenetetrahydrofolate reductase and risk of schizophrenia: a meta-analysis. Mol Psychiatry 2006; 11:143-149.

105 Nishi A, Numata S, Tajima A, Kinoshita M, Kikuchi K, Shimodera S, et al: Meta-analyses of blood homocysteine levels for gender and genetic association studies of the MTHFR C677T polymorphism in schizophrenia. Schizophr Bull 2014;40:1154-1163.

106 Ellingrod VL, Miller DD, Taylor SF, Moline J, Holman T, Kerr J: Metabolic syndrome and insulin resistance in schizophrenia patients receiving antipsychotics genotyped for the methylenetetrahydrofolate reductase (MTHFR) 677C/T and 1298A/C variants. Schizophr Res 2008;98:47-54.

107 Ellingrod VL, Taylor SF, Dalack G, Grove TB, Bly MJ, Brook RD, et al: Risk factors associated with metabolic syndrome in bipolar and schizophrenia subjects treated with antipsychotics: the role of folate pharmacogenetics. J Clin Psychopharmacol 2012;32: 261-265.

108 Devlin AM, Ngai YF, Ronsley R, Panagiotopoulos C: Cardiometabolic risk and the MTHFR C677T variant in children treated with second-generation antipsychotics. Transl Psychiatry 2012;2:e71.

109 Kao AC, Rojnic Kuzman M, Tiwari AK, Zivkovic MV, Chowdhury NI, Medved V, et al: Methylenetetrahydrofolate reductase gene variants and antipsychotic-induced weight gain and metabolic disturbances. J Psychiatr Res 2014;54:36-42.

110 Srisawat U, Reynolds GP, Zhang ZJ, Zhang XR, Arranz B, San L, et al: Methylenetetrahydrofolate reductase (MTHFR) $677 \mathrm{C} / \mathrm{T}$ polymorphism is associated with antipsychotic-induced weight gain in first-episode schizophrenia. Int J Neuropsychopharmacol 2014;17:485-490.
111 van Winkel R, Rutten BP, Peerbooms O, Peuskens J, van Os J, De Hert M: MTHFR and risk of metabolic syndrome in patients with schizophrenia. Schizophr Res 2010; 121:193-198.

112 van Winkel R, Moons T, Peerbooms O, Rutten B, Peuskens J, Claes S, et al: MTHFR genotype and differential evolution of metabolic parameters after initiation of a second generation antipsychotic: an observational study. Int Clin Psychopharmacol 2010;25: 270-276.

113 Marini NJ, Gin J, Ziegle J, Keho KH, Ginzinger D, Gilbert DA, et al: The prevalence of folate-remedial MTHFR enzyme variants in humans. Proc Natl Acad Sci USA 2008; 105 : 8055-8060.

114 Oeschger FM, Wang WZ, Lee S, GarciaMoreno F, Goffinet AM, Arbones ML, et al: Gene expression analysis of the embryonic subplate. Cereb Cortex 2012;22:1343-1359.

115 Brandl EJ, Tiwari AK, Zai CC, Nurmi EL, Chowdhury NI, Arenovich T, et al: Genome-wide association study on antipsychotic-induced weight gain in the CATIE sample. Pharmacogenomics J DOI: 10.1038/ tpj.2015.59.

116 Kennedy JL, Altar CA: Clinical Utility and Enhancements of a Pharmacogenomic Decision Support Tool for Mental Health Patients. Ottawa, GenomeCanada, 2015. http://www.genomecanada.ca/medias/pdf/ en/clinical-utility-improvements-pharmacogenomic-decision-support-tool.pdf.

117 Gough AC, Smith CA, Howell SM, Wolf CR, Bryant SP, Spurr NK: Localization of the CYP2D gene locus to human chromosome 22q13.1 by polymerase chain reaction, in situ hybridization, and linkage analysis. Genomics 1993;15:430-432.

118 Chinta SJ, Pai HV, Upadhya SC, Boyd MR, Ravindranath V: Constitutive expression and localization of the major drug metabolizing enzyme, cytochrome P4502D6 in human brain. Brain Res 2002;103:49-61.

119 Kalman LV, Agundez JA, Appell ML, Black JL, Bell GC, Boukouvala S, et al: Pharmacogenetic Allele Nomenclature: International Workgroup Recommendations for Test Result Reporting. Clin Pharmacol Ther DOI: 10.1002/cpt.280.

120 Bertilsson L, Dahl ML, Dalen P, Al-Shurbaji A: Molecular genetics of CYP2D6: clinical relevance with focus on psychotropic drugs. Br J Clin Pharmacol 2002;53:111-122.

121 Thelma B, Srivastava V, Tiwari AK: Genetic underpinnings of tardive dyskinesia: passing the baton to pharmacogenetics. Pharmacogenomics 2008;9:1285-1306.

122 Kobylecki CJ, Jakobsen KD, Hansen T, Jakobsen IV, Rasmussen HB, Werge T: CYP2D6 genotype predicts antipsychotic side effects in schizophrenia inpatients: a retrospective matched case-control study. Neuropsychobiology 2009;59:222-226.
123 Crescenti A, Mas S, Gasso P, Parellada E, Bernardo M, Lafuente A: CYP2D6*3, *4, *5 and $* 6$ polymorphisms and antipsychoticinduced extrapyramidal side-effects in patients receiving antipsychotic therapy. Clin Exp Pharmacol Physiol 2008;35:807-811.

124 Fleeman N, Dundar Y, Dickson R, Jorgensen A, Pushpakom S, McLeod C, et al: Cytochrome $\mathrm{P} 450$ testing for prescribing antipsychotics in adults with schizophrenia: systematic review and meta-analyses. Pharmacogenomics J 2011;11:1-14.

125 Koola MM, Tsapakis EM, Wright P, Smith S, Kerwin Rip RW, Nugent KL, et al: Association of tardive dyskinesia with variation in CYP2D6: is there a role for active metabolites? J Psychopharmacol 2014;28:665-670.

126 Gasso P, Papagianni K, Mas S, de Bobadilla RF, Arnaiz JA, Bernardo M, et al: Relationship between CYP2D6 genotype and haloperidol pharmacokinetics and extrapyramidal symptoms in healthy volunteers. Pharmacogenomics 2013;14:1551-1563.

127 Gasso P, Mas S, Papagianni K, Ferrando E de Bobadilla RF, Arnaiz JA, et al: Effect of CYP2D6 on risperidone pharmacokinetics and extrapyramidal symptoms in healthy volunteers: results from a pharmacogenetic clinical trial. Pharmacogenomics 2014;15: $17-28$.

128 Locatelli I, Kastelic M, Koprivsek J, KoresPlesnicar B, Mrhar A, Dolzan V, et al: A population pharmacokinetic evaluation of the influence of CYP2D6 genotype on risperidone metabolism in patients with acute episode of schizophrenia. Eur J Pharm Sci 2010; 41:289-298.

129 Tsai HT, Caroff SN, Miller DD, McEvoy J, Lieberman JA, North KE, et al: A candidate gene study of tardive dyskinesia in the CATIE schizophrenia trial. Am J Med Genet B Neuropsychiatr Genet 2010;153B:336340.

130 Jovanovic N, Bozina N, Lovric M, Medved V, Jakovljevic M, Peles AM: The role of CYP2D6 and ABCB1 pharmacogenetics in drug-naive patients with first-episode schizophrenia treated with risperidone. Eur J Clin Pharmacol 2010;66:1109-1117.

131 Tyren M, Arinda E, Connie M, Louw R, Werdie vS, Michael SP: Risperidone-associated adverse drug reactions and CYP2D6 polymorphisms in a South African cohort. Appl Transl Genomic 2015;5:40-46.

132 Seeman P: Atypical antipsychotics: mechanism of action. Can J Psychiatry 2002;47: 27-38.

133 Graybiel AM, Aosaki T, Flaherty AW, Kimura M: The basal ganglia and adaptive motor control. Science 1994;265:18261831.

134 Margolese HC, Chouinard G, Kolivakis TT, Beauclair L, Miller R: Tardive dyskinesia in the era of typical and atypical antipsychotics. 1. Pathophysiology and mechanisms of induction. Can J Psychiatry 2005;50:541-547.
Genetics of Common AntipsychoticInduced Adverse Effects
Mol Neuropsychiatry 2016;2:61-78 DOI: $10.1159 / 000445802$ 
135 Marsden CD, Jenner P: The pathophysiology of extrapyramidal side-effects of neuroleptic drugs. Psychol Med 1980;10:55-72.

136 Zai CC, De Luca V, Hwang RW, Voineskos A, Müller DJ, Remington G, et al: Metaanalysis of two dopamine D2 receptor gene polymorphisms with tardive dyskinesia in schizophrenia patients. Mol Psychiatry 2007;12:794-795.

137 Bakker PR, van Harten PN, van Os J: Antipsychotic-induced tardive dyskinesia and polymorphic variations in COMT, DRD2, CYP1A2 and MnSOD genes: a meta-analysis of pharmacogenetic interactions. Mol Psychiatry 2008;13:544-556.

138 Koning JP, Vehof J, Burger H, Wilffert B, Al Hadithy A, Alizadeh B, et al: Association of two DRD2 gene polymorphisms with acute and tardive antipsychotic-induced movement disorders in young Caucasian patients. Psychopharmacology (Berl) 2012;219:727736.

139 Park YM, Kang SG, Choi JE, Kim YK, Kim SH, Park JY, et al: No evidence for an association between dopamine D2 receptor polymorphisms and tardive dyskinesia in Korean schizophrenia patients. Psychiatry Investig 2011;8:49-54.

140 Zai CC, Tiwari AK, Mazzoco M, de Luca V, Müller DJ, Shaikh SA, et al: Association study of the vesicular monoamine transporter gene SLC18A2 with tardive dyskinesia. J Psychiatr Res 2013;47:1760-1765.

141 Bishop JR, Schuksz M, Esko JD: Heparan sulphate proteoglycans fine-tune mammalian physiology. Nature 2007;446:10301037.

142 Nishida T, Kubota S, Fukunaga T, Kondo S, Yosimichi G, Nakanishi T, et al: CTGF/ Hcs24, hypertrophic chondrocyte-specific gene product, interacts with perlecan in regulating the proliferation and differentiation of chondrocytes. J Cell Physiol 2003;196: 265-275.
143 Fuki II, Iozzo RV, Williams KJ: Perlecan heparan sulfate proteoglycan. A novel receptor that mediates a distinct pathway for ligand catabolism. J Biol Chem 2000;275: 31554.

144 Inada T, Koga M, Ishiguro H, Horiuchi $\mathrm{Y}$, Syu A, Yoshio T, et al: Pathway-based association analysis of genome-wide screening data suggest that genes associated with the gamma-aminobutyric acid receptor signaling pathway are involved in neuroleptic-induced, treatment-resistant tardive dyskinesia. Pharmacogenet Genomics 2008;18:317323.

145 Syu A, Ishiguro H, Inada T, Horiuchi Y, Tanaka S, Ishikawa M, et al: Association of the HSPG2 gene with neuroleptic-induced tardive dyskinesia. Neuropsychopharmacology 2010;35:1155-1164.

146 Greenbaum L, Alkelai A, Zozulinsky P, Kohn Y, Lerer B: Support for association of HSPG2 with tardive dyskinesia in Caucasian populations. Pharmacogenomics J 2012;12: 513-520.

147 Bakker PR, Al Hadithy AF, Amin N, van Duijn CM, van Os J, van Harten PN: Antipsychotic-induced movement disorders in long-stay psychiatric patients and $45 \mathrm{tag}$ SNPs in 7 candidate genes: a prospective study. PLoS One 2012;7:e50970.

148 Lin L, Long LK, Hatch MM, Hoffman DA: DPP6 domains responsible for its localization and function. J Biol Chem 2014;289: 32153-32165.

149 Tanaka S, Syu A, Ishiguro H, Inada T, Horiuchi Y, Ishikawa M, et al: DPP6 as a candidate gene for neuroleptic-induced tardive dyskinesia. Pharmacogenomics J 2013;13: 27-34.
150 Emond MJ, Louie T, Emerson J, Zhao W, Mathias RA, Knowles MR, et al: Exome sequencing of extreme phenotypes identifies DCTN4 as a modifier of chronic Pseudomonas aeruginosa infection in cystic fibrosis. Nat Genet 2012;44:886-889.

151 Lanktree MB, Hegele RA, Schork NJ, Spence JD: Extremes of unexplained variation as a phenotype: an efficient approach for genome-wide association studies of cardiovascular disease. Circ Cardiovasc Genet 2010;3 215-221.

152 Cordell HJ: Detecting gene-gene interactions that underlie human diseases. Nat Rev Genet 2009;10:392-404.

153 Lee S, Kim Y, Kwon MS, Park T: A comparative study on multifactor dimensionality reduction methods for detecting gene-gene interactions with the survival phenotype. Biomed Res Int 2015;2015:671859.

154 Li J, Huang D, Guo M, Liu X, Wang C, Teng $Z$, et al: A gene-based information gain method for detecting gene-gene interactions in case-control studies. Eur J Hum Genet DOI: 10.1038/ejhg.2015.16.

155 Jiang X, Neapolitan RE: LEAP: biomarker inference through learning and evaluating association patterns. Genet Epidemiol 2015; 39:173-184.

156 Lan TH, Loh EW, Wu MS, Hu TM, Chou P, Lan TY, et al: Performance of a neuro-fuzzy model in predicting weight changes of chronic schizophrenic patients exposed to antipsychotics. Mol Psychiatry 2008;13: 1129-1137.

157 Maranville JC, Cox NJ: Pharmacogenomic variants have larger effect sizes than genetic variants associated with other dichotomous complex traits. Pharmacogenomics J DOI: 10.1038/tpj.2015.47. 